\title{
UNA NUEVA ESPECIE DE MELOE LINNAEUS, 1758 DEL SUROESTE DE MARRUECOS INCLUIDA EN EL GRUPO DE M. (EURYMELOE) RUGOSUS MARSHAM, 1802 (COLEOPTERA: MELOIDAE)
}

\author{
José L. Ruiz* \& M. García-París**,† \\ * Instituto de Estudios Ceuties, Paseo del Revellin, 30.51001 Ceuta, España. E-mail: euserica@hotmail.com \\ urn:Isid:zoobank.org:author:D633356A-58DA-442D-B726-F3EF7B53D4BF \\ ** Museo Nacional de Ciencias Naturales (MNCN-CSIC), c/José Gutiérrez \\ Abascal, 2. 28006 Madrid, España. E-mail: mparis@mncn.csic.es \\ urn:Isid:zoobank.org:author:5FD2079F-52BF-41F4-8C88-70593E306DA0
}

${ }^{+}$Autor para correspondencia

\section{RESUMEN}

Las colecciones científicas del Museo Nacional de Ciencias Naturales (MNCN-CSIC, Madrid) albergan un extenso material entomológico recogido en Marruecos a principios del siglo XX por el insigne naturalista M. Martínez de la Escalera. El estudio morfológico del material del género Meloe Linnaeus, 1758 (Coleoptera: Meloidae) revela la existencia de poblaciones morfológicamente diferenciadas en las regiones costeras de Essaouira e Ifni. Estas poblaciones se encuadran en el grupo de Meloe rugosus Marsham, 1802 dentro del subgénero Eurymeloe Reitter, 1911. Sus características diferenciales y constantes respecto al resto de las especies norteafricanas y europeas del grupo, permiten tratar a este conjunto de poblaciones como una entidad taxonómicamente independiente que se describe aquí, Meloe baamarani n. sp. Esta especie nueva se caracteriza por presentar la coloración del cuerpo y apéndices negra, de aspecto mate; pilosidad negra, corta; cabeza ancha y de sienes ampliamente redondeadas, sin surco longitudinal medio; antenas largas, con los artejos III a VIII más largos que anchos, subcilíndricos; pronoto transverso, con los lados netamente convergentes hacia atrás, sin surco mediano; punteado de cabeza y pronoto denso; edeago estrecho, lóbulo medio largo y robusto, sinuado dorsalmente, con los dientes ventrales próximos al ápice. En la región occidental del noroeste africano $M$. baamarani puede confundirse únicamente con Meloe mediterraneus Müller, 1925, especie con la que comparte un aspecto general similar, pero de la que se diferencia por numerosos caracteres morfológicos, entre los que se incluyen la microrreticulación del tegumento, la ausencia de surco medio en la cabeza, la morfología y macroescultura del pronoto, y la configuración de la genitalia masculina.

urn:Isid:zoobank.org:pub:D5A8AFFA-D7E9-4E65-8E73-C6AC58AF20B6

Palabras clave: Coleoptera; Meloidae; Meloe; Eurymeloe; grupo de especies de Meloe rugosus; especie nueva; Marruecos; norte de África; Taxonomía; Biodiversidad.

\section{ABSTRACT}

A new species of Meloe Linnaeus, 1758 from southwestern Morocco included in the M. (Eurymeloe) rugosus Marsham, 1802 species group (Coleoptera: Meloidae)

The scientific collections of the Museo Nacional de Ciencias Naturales (MNCN-CSIC, Madrid) hold an extense set of entomological materials collected in Morocco along the first decades of the XXth century by the preeminent naturalist M. Martínez de la Escalera. Morphological studies of the specimens of the genus Meloe Linnaeus, 1758 (Coleoptera: Meloidae) reveals the existence of populations morphologically differentiated along the coastal regions of Essaouira and Ifni. These populations are included within the Meloe rugosus Marsham, 1802 species group in the subgenus Eurymeloe Reitter, 1911. Their differential traits with respect to all other North African and European species of the Meloe rugosus species group are constant, and permit considering these populations as a taxonomic independent unit described herein, Meloe baamarani $\mathbf{n}$. sp. This new species is characterized by having a black, opaque, general coloration all over the body and appendages; black short vestiture; broad head with broadly rounded temples, without median longitudinal groove; long antennae, with segments III to VIII subcylindrical and longer than wide; pronotum transverse, with convergent sides toward the base, without median groove; head and pronotum punctuation dense; aedeagus narrow, median lobe wide and strong, 


\begin{abstract}
dorsally sinuous, with ventral hooks close to the apex. Meloe baamarani can be only confused on the western regions of northern Africa with Meloe mediterraneus Müller, 1925. This species shares a general appearance with $M$. baamarani, but differs in many morphological traits. Among those, tegument micro-reticulation, absence of median groove along the head, pronotum morphology and macrosculpture, and configuration of the male genitalia, are included.
\end{abstract}

Key words: Coleoptera; Meloidae; Meloe; Eurymeloe; Meloe rugosus species-group; new species; Morocco; northern Africa; Taxonomy; Biodiversity.

\title{
Recibido/Received: 05/08/2014; Aceptado/Accepted: 26/01/2015; Publicado en línea/Published online: 18/03/2015
}

Cómo citar este artículo/Citation: Ruiz, J. L. \& García-París, M., 2015. Una nueva especie de Meloe Linnaeus, 1758 del suroeste de Marruecos incluida en el grupo de M. (Eurymeloe) rugosus Marsham, 1802 (Coleoptera: Meloidae). Graellsia, 71(1): e018. http://dx.doi.org/10.3989/graellsia.2015.v71.118.

Copyright: (C) 2015 SAM y CSIC. Salvo indicación contraria, todos los contenidos de la edición electrónica de Graellsia se distribuyen bajo licencia de uso y distribución Creative Commons Reconocimiento no Comercial 3.0. España (cc-by-nc).

\section{Introducción}

El género Meloe Linnaeus, 1758 agrupa a unas 155 especies integradas en 16 subgéneros, y presenta una amplia distribución por las regiones templadas de la tierra (Pinto \& Selander, 1970; Bologna, 1991; Bologna $\&$ Pinto, 2002). Entre el elevado número de subgéneros válidos actualmente reconocidos, Eurymeloe Reitter, 1911 [especie-tipo: Meloe brevicollis Panzer, 1793, por designación posterior de Pinto \& Selander (1970)] es uno de los más diversos y complejos. En efecto, se trata de un taxón morfológicamente muy heterogéneo, de dudosa monofilia, que integra en su concepción actual a unas 50 especies de distribución mayoritariamente paleártica (e.g. Bologna, 1988, 1991, 2008; Bologna et al., 1989; Ruiz \& García-París, 2009; Ruiz et al., 2010; Di Giulio et al., 2013).

Atendiendo a determinados caracteres morfológicos y desde un prisma eminentemente práctico, Bologna (1988) definió para Eurymeloe dos grandes grupos bien diferenciados: grupo I o grupo de Meloe rugosus Marsham, 1802, que integra unas 25 especies caracterizadas por su color negro, tamaño mediopequeño, pilosidad negra o amarillo-castaña y antenas con artejos normalmente alargados y subcilíndricos, de lados paralelos; grupo II o grupo de Meloe brevicollis Panzer, 1793, que agrupa en torno a otras 24 especies, caracterizadas por su tamaño mayor, coloración corporal azulada o negra, pilosidad muy reducida $\mathrm{y}$ artejos de las antenas poco alargados, generalmente subcónicos (Bologna, 1988, 1991, 2008).

A su vez, dentro del grupo I o de $M$. rugosus, este autor estableció dos subgrupos (Bologna 1988): el subgrupo A, con la pilosidad corporal negra (en ciertos casos, en parte castaño-amarillenta) y el tegumento corporal negro brillante u opaco, con 15 especies; y el subgrupo B, o de Meloe murinus Brandt \& Erichson, 1832 , diferenciado del anterior fundamentalmente por presentar la pilosidad clara (amarillenta) y el tegumento negro-grisáceo mate, habitualmente con las patas y antenas en parte de color castaño (Bologna, 1988, 1991, 1994a). Por otra parte, Ruiz et al. (2010) proponen un nuevo subgrupo dentro del grupo I, para incluir a las especies del complejo de $M$. saharensis Chobaut, 1898, muy distintas morfológicamente a las de los otros dos subgrupos.

Recientemente, Di Giulio et al. (2013), a partir del estudio comparado de las larvas de primer estadio de varias especies de Eurymeloe, concluyen que la morfología larvaria no apoya la subdivisón propuesta por Bologna (1988), pero no proponen una hipótesis de conjunto alternativa.

Con anterioridad a la revisión de las especies occidentales del grupo de $M$. rugosus efectuada por Bologna (1988), buena parte de los taxones específicos se encontraban confundidos y eran interpretados de forma diversa e incluso contradictoria. En el caso del subgrupo A, una especie mal interpretada de forma recurrente era el propio M. rugosus. La distribución real de esta especie es europeo-turánica, pero fue erróneamente citado por numerosos autores de toda la región mediterránea, incluido el norte de África y la totalidad de las penínsulas ibérica e italiana (ver referencias en Bologna, 1988, 1991, 1994b, 2008; GarcíaParís \& Ruiz, 2005). La inmensa mayoría de estas citas antiguas se corresponden realmente a otras especies de distribución más meridional, como $M$. affinis Lucas, 1847, exclusivamente magrebí, $M$. mediterraneus Müller, 1925, ampliamente difundida por toda la cuenca mediterránea (incluido el Magreb) o, en el caso concreto de Italia, M. apenninicus Bologna, 1988, M. ganglbaueri Apfelbeck, 1907 o M. baudii Leoni, 1907 (véase Bologna, 1988, 1991, 1994b).

De igual forma, el complejo de $M$. affinis, actualmente con cuatro subespecies reconocidas, M. a. affinis, M. a. simillimus Martínez de la Escalera, 1914, M. a. apivorus Reitter, 1895 y M. a. schatzmayri Bologna, 1988 (ver Bologna, 1988, 1991, 2008, 2009; García-París \& Ruiz, 2011), también ha sido diversamente interpretado, y tales subespecies 
han sido indistintamente tratadas como variedades o como especies válidas (e.g. Escherich, 1890, 1896; Reitter, 1895; Martínez de la Escalera, 1914; Borchmann, 1917; Mader, 1927; Cros, 1934, 1936, 1939; Peyerimhoff, 1926, 1949; Kocher, 1953, 1956). Recientemente, Di Giulio et al. (2013), basándose en el estudio de caracteres larvarios, consideran que $M$ apivorus sería una especie válida, conocida hasta el momento únicamente del Alto Atlas suroccidental (Tizi-n-Test).

Evidentemente, la representación marroquí de Eurymeloe no se sustrajo de la confusión imperante en la taxonomía interna de este subgénero (e.g. Ruiz \& García-París, 2009; Ruiz et al., 2010), siendo especialmente problemáticos los casos de $M$. rugosus y M. affinis. Así, en Marruecos M. rugosus fue citado por Martínez de la Escalera (1914) de la región de Tánger. Sin embargo, Kocher (1956: 69, nota 3), siguiendo el parecer de Müller (1925) y Peyerimhoff (in litt.), asumió que los registros marroquíes de $M$. rugosus debían considerarse obsoletos y habrían de referirse a M. mediterraneus [citada como "M. (rugosus Marsh.) var. mediterraneus G. Muell."], a la que atribuyen una distribución por todo el norte, occidente y centro de Marruecos. Posteriormente, Bologna (1988, 1991) refrenda el criterio de Kocher (1956), excluyendo a $M$. rugosus de la fauna marroquí.

De igual forma, el complejo de $M$. affinis (Bologna, 1988, 1991, 2008, 2009; García-París \& Ruiz, 2011) también ha sido diversamente interpretado, y las diferentes entidades que lo componen han sido indistintamente tratadas como variedades o como especies válidas (e.g. Escherich, 1890, 1896; Reitter, 1895; Martínez de la Escalera, 1914; Borchmann, 1917; Mader, 1927; Cros, 1934, 1936, 1939; Peyerimhoff, 1926, 1949; Kocher, 1953, 1956). Meloe affinis es una especie altamente variable, cuya taxonomía interna se encuentra todavía lejos de solución (Di Giulio et al., 2013) y requiere una profunda revisión de conjunto. Bologna (1988, 1991, 2008, 2009; aunque ver también Di Giulio et al., 2013) reconoce al menos cuatro subespecies dentro de M. affinis: M. a. affinis que se distribuye por el norte de Argelia y Túnez, y cuya presencia en el norte de Marruecos debe confirmarse; M. a. simillimus Martínez de la Escalera, 1914, descrita como especie válida, propia del Medio Atlas y del Alto Atlas occidental, núcleos montañosos del centro y suroeste de Marruecos, de donde se conoce de escasas localidades (Kocher, 1956; Bologna, 1988, 1991); M. a. apivorus Reitter, 1895, también descrita como especie independiente a partir de un único ejemplar hembra de "Marokko" (loc. typ.) sin localidad precisa (Reitter, 1895); y por último, M. a. schatzmayri Bologna, 1988, endémica de Libia (Tripolitania) (Bologna, 1988, 1991, 2008, 2009).

Meloe affinis fue citado por Martínez de la Escalera (1914) en Marruecos de Mogador (=Essaouira) en la costa suratlántica, y de Tifnut (=Tifnout) y Tizi-n-Test en el Alto Atlas occidental; sin embargo, tanto Kocher (1956: 70) como Bologna (1988: 269) coinciden en que el registro de Mogador de $M$. affinis (sin distinción de subespecie o variedad) ofrecido por Martínez de la Escalera (1914: 383) es dudoso, e incluso el primero de ellos afirma (sic): "Les localités indiqées par Escalera (Mogador, etc.) sont a considérer comme erronées; elles ne figurent d'ailleurs pas dans sa collection où tous les exemplaires de affinis sont du Moyen-Atlas" (Kocher, 1956: 70, nota 1). ¿A qué taxon correspondería entonces la población de Meloe de Mogador referida por M. Martínez de la Escalera como M. affinis?.

A fecha actual son dos las especies de Eurymeloe del subgrupo A del grupo de M. rugosus presentes en Marruecos: M. mediterraneus y $M$. affinis, esta última representada por uno o dos taxones subespecíficos o específicos, M. a. simillimus y M. a. apivorus, según el criterio considerado (Bologna, 1988, 1991; Di Giulio et al., 2013). Sin embargo, la atribución taxonómica de los ejemplares citados por Martínez de la Escalera (1914) sólo puede realizarse tras el examen de los mismos o de otros de idéntica procedencia.

La revisión de material marroquí de Eurymeloe de las colecciones históricas del Museo Nacional de Ciencias Naturales, Madrid (CSIC, España) nos ha permitido localizar los ejemplares de Mogador citados por Martínez de la Escalera (1914) como M. affinis [contrariamente a lo expresado por Kocher (1956: 70)], así como material morfológicamente similar procedente del territorio de Ifni. Tras el estudio comparado de estos ejemplares y abundante material de las otras especies mediterráneas del subgrupo A del grupo de M. rugosus, consideramos que se trata de una especie inédita, cuya descripción constituye el objeto de este trabajo.

\section{Material y métodos}

La descripción de la especie nueva de Meloe se basa en un total de 18 ejemplares, 11 machos y 7 hembras, conservados en seco en la colección de entomología del Museo Nacional de Ciencias Naturales (MNCN-CSIC) (Madrid, España). Dicho material procede del Marruecos suratlántico, concretamente de Mogador (actual Essaouira) y de varias localidades del antiguo territorio de Ifni. Los ejemplares de Mogador provienen de las capturas del entomólogo español Manuel Martínez de la Escalera y Pérez de Rozas (1867-1949) durante sus primeras estancias en esta localidad costera del suroeste de Marruecos (años 1905 y 1906), donde permaneció de forma más o menos continua entre 1905-1911, en calidad de Secretario de la "Comisión para la exploración y estudio del Noroeste de África", auspiciada por la Real Sociedad Española de Historia Natural y 
financiada tanto por ésta como por el estado español (Martínez de la Escalera, 1914; Martín Albaladejo, 2011; Pina, 2011). Los ejemplares del territorio de Ifni (entre ellos, el designado como holotipo) son fruto de las colectas de Fernando Martínez de la Escalera Goróstegui (1895-1988, hijo de Manuel) en una expedición llevada a cabo entre diciembre de 1934 y enero de 1935 (Bolívar, 1935; Martínez de la Escalera y Carrasco, 2011), en aquellos territorios entonces bajo dominio español (aproximadamente, 2000 km²; véase e.g. García-Figueras, 1941).

El holotipo y todos los paratipos de la nueva especie se encuentran depositados en el MNCN. Como material de comparación de los taxones específicos del subgrupo en el que se integra la nueva especie (subgrupo A del grupo de M. rugosus Marsham, 1802), se han estudiado un total de 162 ejemplares de las especies presentes en la región mediterránea occidental y macaronésica que a continuación se indican y cuyos datos se relacionan en el Apéndice 1 (entre ellos, un sintipo de Meloe simillimus Martínez de la Escalera, 1914, véase García-París \& Ruiz, 2011, y un paratipo de Meloe apenninicus, véase Bologna, 1988): M. rugosus Marsham, 1802 (4 ejemplares), $M$. apenninicus Bologna, 1988 (3 ejemplares), M. baudii Leoni, 1907 (1 ejemplar), M. ganglbaueri Apfelbeck, 1905 (7 ejemplares), M. affinis simillimus Martínez de la Escalera, 1914 (29 ejemplares), M. affinis apivorus Reitter, 1895 (2 ejemplares), M. mediterraneus Müller, 1925 (108 ejemplares) y M. fernandezi Pardo Alcaide, 1951 (8 ejemplares). Las instituciones y colecciones de las que se ha estudiado material de comparación, y sus respectivas siglas, son las siguientes: Museo Nacional de Ciencias Naturales-CSIC, Madrid, España $(\mathrm{MNCN})$; Estación Experimental de Zonas Áridas-CSIC, Almería, España (EEZA); Museu de Zoologia, Barcelona, España (MZB); Muséum National d'Histoire Naturelle, París, Francia (MNHN); Universidad de La Laguna, Tenerife, España (UL); colección M.A. Bologna, en la Universidad de "Roma Tre", Roma, Italia (MB); colección J.L. Ruiz, Ceuta, España (JLR); colección Ramón Sánchez, España (RS); colección José Luis Zapata, Madrid, España (JLZ); y colección Thomas Gazurek, Polonia (TG).

El estudio morfológico se efectuó sobre ejemplares conservados en seco, mediante observación con estereomicroscopio. El aparato genital masculino, extraído a ejemplares secos previamente rehidratados, se montó en cartulinas con resina DMHF, que se pincharon con su respectivo ejemplar. Las medidas se efectuaron con micrómetro acoplado a uno de los oculares y los dibujos con cámara clara. La longitud total de los ejemplares se ha tomado desde el extremo de la frente (en posición dorsal) hasta el extremo del abdomen. La medida de la longitud máxima del pronoto se ha tomado desde el nivel del extremo del lóbulo del margen posterior hasta extremo del ángulo anterior (Fig. 1); la medida de la anchura pronotal corresponde

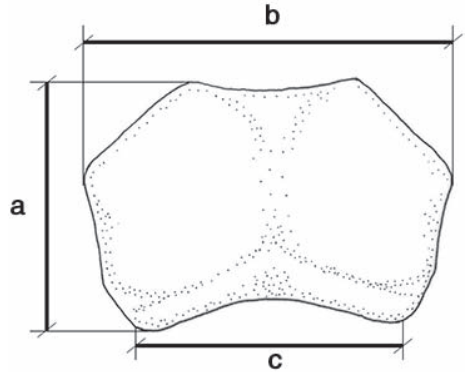

Fig. 1.- Pronoto esquemático de Eurymeloe en el que se muestran las medidas tomadas: (a) longitud; (b) anchura; (c) anchura en la base.

Fig. 1.- Schematic design of the pronotum of Eurymeloe showing the measurements taken: (a) length; (b) width; (c) with at the base.

a la máxima entre los márgenes laterales, es decir, la máxima observada en visión dorsal; y la medida de la "anchura del pronoto en la base" es la tomada entre los respectivos puntos de inflexión de los márgenes laterales en la base. Las medidas pronotales de la serie-tipo de la nueva especie se obtuvieron de 17 de los 18 ejemplares que la componen, debido a que un ejemplar hembra procedente de Ain Agisgal (Ifni) presenta el pronoto deformado (teratología), totalmente estrangulado en el centro, con los ángulos anteriores y posteriores desplazados, no siendo representativo.

Para determinar la relación "longitud/anchura máxima" en los artejos antenales de la nueva especie respecto a $M$. a. simillimus y $M$. a. apivorus, se consideró como representativa la correspondiente a los artejos IV a VII de la antena derecha, de los cuales se tomaron las correspondientes medidas en 13 ejemplares de la serie-tipo (en 5 paratipos no se obtuvieron estas medidas, a fin de no dañar los ejemplares con una manipulación innecesaria), en 26 de $M$. $a$. simillimus y en 2 de $M$. a. apivorus. Las fotografías se efectuaron con cámara digital. La terminología de las diversas partes de la genitalia masculina se basa en la expuesta por Bologna $(1988,1991)$.

Los estadios larvarios de la nueva especie son desconocidos, por lo que la discusión sobre la posición taxonómica y afinidades se efectúa exclusivamente a partir de caracteres morfológicos de los adultos. Hemos adoptado el esquema taxonómico propuesto por Bologna (1988), dada su evidente utilidad práctica para diferenciar grupos de especies dentro de Eurymeloe mediante el uso de caracteres morfológicos imaginales, aunque las agrupaciones resultantes pudieran no corresponderse con entidades monofiléticas (Di Giulio et al., 2013). De igual forma, respecto a la taxonomía interna del complejo de $M$. affinis, y a efectos meramente prácticos, se sigue la expuesta por Bologna (1988, 1991, 2008, 2009).

El concepto de especie adoptado en este artículo, que es el utilizado implícitamente por los autores en otros trabajos, es el "concepto evolutivo" (modificado 
de Wiley, 1978, 1981 y Wiley \& Mayden, 2000), en el que básicamente, una especie puede definirse como un linaje de poblaciones único que mantiene su identidad respecto a otros linajes y que tiene sus propias tendencias evolutivas y devenir histórico. Este concepto combina las implicaciones metodológicas básicas del concepto filogenético de especie, en el que los linajes se definen objetivamente por la existencia de monofilia recíproca, con aspectos subjetivos que permiten caracterizar como unidad independiente la línea filética definida como especie. Este segundo aspecto, que básicamente supone la propuesta de una hipótesis informada sobre el futuro evolutivo del linaje, es crítico para considerar un linaje independiente como especie o no (García-París et al., 2008). Las implicaciones de este concepto suponen que las propuestas taxonómicas han de considerarse como hipótesis evolutivas, en las que el autor propone, por una parte, que la especie nueva está compuesta por un grupo de poblaciones recíprocramente monofilético con respecto a su especie hermana (y por lo tanto con caracteres morfológicos y genéticos diferenciales) (Alcobendas et al., 2008), y por otra que con el devenir histórico existen, por ejemplo, pocas probabilidades de que la nueva especie deje de ser monofilética al hibridar extensivamente con su especie hermana (es decir, el tiempo transcurrido desde su aislamiento es suficiente como para garantizar un cierto grado de aislamiento reproductivo con respecto a su especie hermana) (García-París et al., 2003).

\section{Resultados}

\section{Meloe (Eurymeloe) baamarani Ruiz \& García-París} n. sp.

(Figs. 2-7)

urn:1sid:zoobank.org:act:22018034-AE81-4932-9E295F51C38A8062

Material estudiado: HOLOTYPUS: 1 macho (conservado en seco), etiquetado: "Tazila/(Ifni)/I-1935, F. Escalera" (etiqueta blanca, impresa); "Holotypus/Meloe (Eurymeloe) baamaranil Ruiz \& García-París des. 2015" (etiqueta roja, impresa). En la colección del MNCN (MNCN cat. Tipos 2557).

PARATYPI: 10 machos y 7 hembras (conservados en seco), etiquetados: 2 machos, 1 hembra: "Marruecos/Mogador/XII1905-Escalera" (etiqueta blanca, impresa; en dos de estas etiquetas el número romano original impreso fue corregido a mano con tinta, de forma que a sendas etiquetas impresas con el número "VII" se le añadieron dos líneas oblicuas en el vértice de la V, transformándolo en "XII", y a una tercera etiqueta, de uno de los machos, parece que se le añadieron esas dos líneas débilmente con lápiz); 1 macho, 1 hembra: "Marruecos/Mogador/XII-1906Escalera" (etiqueta blanca, impresa); 3 machos: "Taraut de Tual/ (Ifni)/I-1935, F. Escalera" (etiqueta blanca, impresa); 1 macho: "Adai/(Ifni)/I-1935, F. Escalera" (etiqueta blanca, impresa); 1 macho: "Amasin/(Ifni)/I-1935, F. Escalera" (etiqueta blanca, impresa); 1 macho: "Tazila/(Ifni)/I-1935, F. Escalera" (etiqueta blanca, impresa); 2 machos, 2 hembras: "Ain Aguisgal, / (Ifni)/I-1935, F. Escalera" (etiqueta blanca, impresa); 1 macho,
1 hembra: "Yebel Tamarrut/(Ifni)/I-1935, F. Escalera" (etiqueta blanca, impresa). Todos los paratipos portan la siguiente etiqueta: "Paratypus/Meloe (Eurymeloe) baamarani/Ruiz \& García-París des. 2015" (etiqueta roja, impresa). Todos en la colección del MNCN (MNCN cat. Tipos 2557).

DESCRIPCIÓN DEL HOLOTIPO: Longitud total: $16.1 \mathrm{~mm}$. Anchura máxima, situada en el ápice de los élitros: $8.9 \mathrm{~mm}$. Aspecto general relativamente robusto, aunque con antenas y patas más bien estrechas y alargadas (Figs. 2, 3). Color general del cuerpo negro, mate, únicamente con los artejos de las antenas VII a XI tornándose castaño oscuro, al igual que el último artejo de los palpos maxilares, las espinas tibiales y las uñas. La superficie tegumentaria en la cabeza, pronoto y élitros es fuertemente microrreticulada, lo que condiciona el aspecto mate del tegumento corporal. Pilosidad en su práctica totalidad negra, cortísima y fina, especialmente la de la cabeza, antenas, pronoto, élitros y terguitos abdominales, más larga la del clípeo, labro, patas y esternitos abdominales, sin formar mechones o agrupaciones de pelos; únicamente castaño rojiza la del cuello, que es un poco más larga y erecta que la de la cabeza, al igual que la del mesosternon.

Cabeza grande, voluminosa, ligeramente más ancha que el pronoto, con tegumento negro opaco, mate y fuertemente microrreticulado, con las sienes ampliamente redondeadas y apenas dilatadas, débilmente truncada por detrás (Fig. 4); anchura máxima en visión frontal: $3.85 \mathrm{~mm}$, a nivel del declive de las sienes, un poco por encima del borde superior de los ojos; anchura mínima entre los ojos: $2.05 \mathrm{~mm}$; distancia entre la sutura clípeo frontal y el vértex

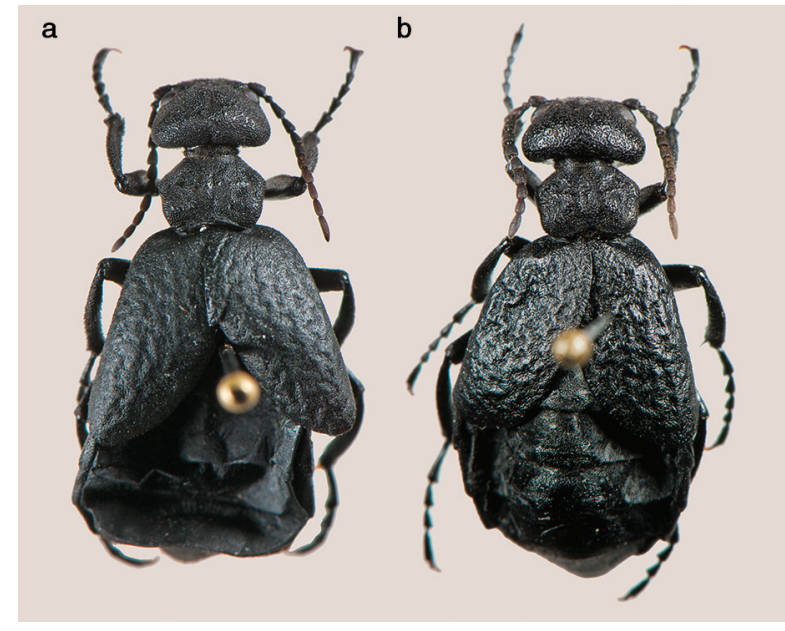

Fig. 2.- Habitus (vista dorsal) de: (a) Meloe (Eurymeloe) baamarani n. sp. (Holotipo: Tazila, Ifni, Marruecos) (longitud del ejemplar: $16.2 \mathrm{~mm}$ ); (b) Meloe (Eurymeloe) mediterraneus Müller, 1925 (Sierra del Haus, Tetuán, Marruecos) (longitud del ejemplar: $16.3 \mathrm{~mm}$ ) (Fotografías: Saúl Yubero).

Fig. 2.- Habitus (dorsal view) of: (a) Meloe (Eurymeloe) baamarani n. sp. (Holotype: Tazila, Ifni, Morocco) (specimen length: $16.2 \mathrm{~mm}$ ); (b) Meloe (Eurymeloe) mediterraneus Müller, 1925 (Sierra del Haus, Tetouan, Morocco) (specimen length: $16.3 \mathrm{~mm}$ ) (Photographs: Saúl Yubero). 


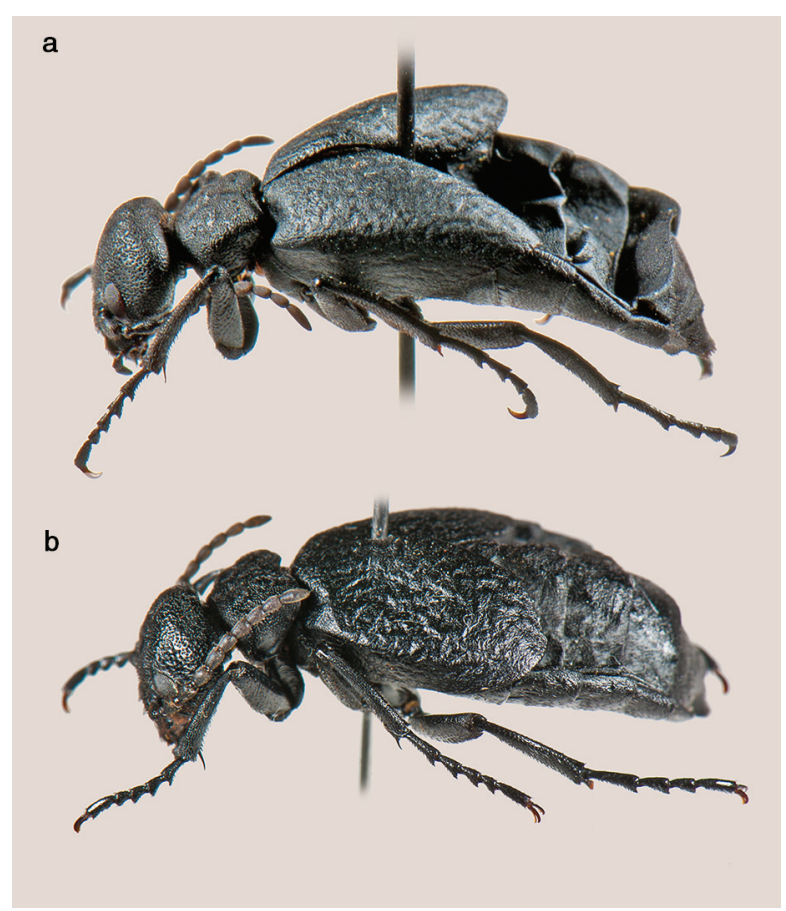

Fig. 3.- Habitus (vista lateral) de: (a) Meloe (Eurymeloe) baamarani n. sp. (Holotipo: Tazila, Ifni, Marruecos) (longitud del ejemplar: $16.2 \mathrm{~mm}$ ); (b) Meloe (Eurymeloe) mediterraneus Müller, 1925 (Sierra del Haus, Tetuán, Marruecos) (longitud del ejemplar: $16.3 \mathrm{~mm}$ ) (Fotografías: Saúl Yubero).

Fig. 3.- Habitus (lateral view) of: (a) Meloe (Eurymeloe) baamarani n. sp. (Holotype: Tazila, Ifni, Morocco) (specimen length: $16.2 \mathrm{~mm}$ ); (b) Meloe (Eurymeloe) mediterraneus Müller, 1925 (Sierra del Haus, Tetouan, Morocco) (specimen length: $16.3 \mathrm{~mm}$ ) (Photographs: Saúl Yubero).

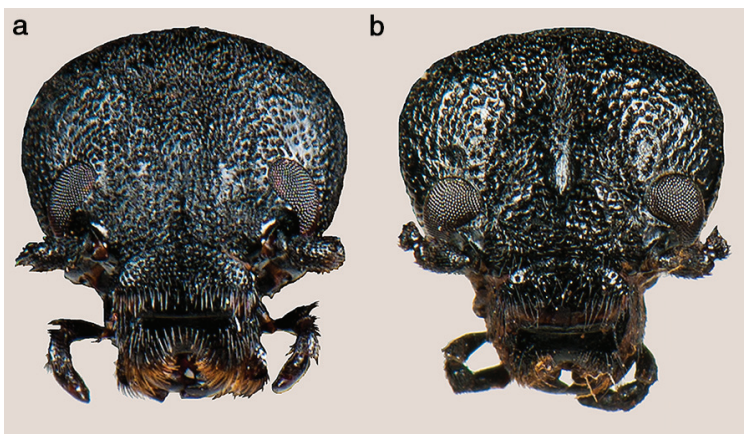

Fig. 4.- Cabeza (vista frontal) de: (a) Meloe (Eurymeloe) baamarani n. sp. (Holotipo: Tazila, Ifni, Marruecos); (b) Meloe (Eurymeloe) mediterraneus Müller, 1925 (Sierra del Haus, Tetuán, Marruecos). Longitud de la cabeza, de la sutura clípeo-frontal al vértex en la línea media, en (a): $2.55 \mathrm{~mm}$; en (b): $2.75 \mathrm{~mm}$. (Fotografías: Saúl Yubero).

Fig. 4.- Head (frontal view) of: (a) Meloe (Eurymeloe) baamarani n. sp. (Holotype: Tazila, Ifni, Morocco); (b) Meloe (Eurymeloe) mediterraneus Müller, 1925 (Sierra del Haus, Tetouan, Morocco). Head length, from fronto-clypeal suture to vertex along the median line of (a): $2.55 \mathrm{~mm}$; and (b): $2.75 \mathrm{~mm}$. (Photographs: Saúl Yubero).

(en visión frontal): $2.65 \mathrm{~mm}$; sin surco longitudinal medio patente y bien definido, en su caso una leve y superficial depresión de corto recorrido (no alcanza el vértex), de límites difusos, un poco por encima del nivel del borde superior de los ojos. Frente casi plana en el disco, sin depresión o surco central, y con sendas depresiones netas, redondeadas, por encima de las inserciones antenales que son marcadamente elevadas. Ojos pequeños, relativamente protuberantes, arriñonados, aunque muy escasamente escotados a nivel de las inserciones antenales, con el lóbulo superior en torno a 1.5 veces más ancho que el inferior. Sutura clípeofrontal neta, pero muy fina y poco profunda, arqueada. Clípeo negro mate, transverso, 2.15 veces más ancho que largo, con los lados rectos, plano en la mayor parte de su superficie, con el tercio anterior membranoso de coloración castaño oscura. Labro superior negro, con los extremos tornándose castaño oscuro, 2.6 veces más ancho que largo, profundamente hendido en el centro, formando dos lóbulos redondeados a ambos lados de la escotadura central. Punteado de la cápsula cefálica en su conjunto denso, constituido por puntos redondos, pequeños, netos y bien impresos, ligeramente variables en tamaño por zonas: subcontiguos en el vértex y en el disco de la frente, donde alcanzan la mayor densidad y menor tamaño, y menos densos en las sienes y por encima de los ojos, donde muestran un diámetro algo mayor. Punteado del clípeo formado por puntos similares a los de la frente, densos, subconfluentes, distribuidos por toda su superficie a excepción de la banda membranosa distal; el del labro similar al del clípeo y restringido a los dos tercios anteriores, concentrado sobre todo en los lóbulos, con el tercio basal apenas punteado. Pilosidad de la cabeza cortísima, negra, semicurvada, en general semitendida hacia atrás en toda la frente y hacia delante en el vértex desde la zona de inflexión del mismo, siguiendo el patrón de distribución del punteado en el que se inserta. Pilosidad del clípeo y labro entre tres y cuatro veces más larga que la de la cápsula cefálica, inclinada hacia delante, igualmente siguiendo el patrón de distribución del punteado. Mandíbulas negras, con los extremos castaño oscuro, mate, de conformación normal, con la mitad posterior de su margen externo densamente punteado-piloso, con puntos y pelos negros un poco más cortos que los del labro. Palpos maxilares alargados, semilustrosos, con el artejo distal castaño oscuro, subcilíndrico y truncado en el ápice, que muestra una pilosidad escasa y más corta que la del labro. Palpos labiales castaño oscuros, casi negros, cortos, con el artejo distal securiforme, muy dilatado. Cuello negro, con punteado denso constituido por puntos un poco más pequeños que los del vértex y pilosidad semierecta, castaño rojiza.

Antenas con once artejos, de aspecto relativamente gráciles, alargadas, extendidas hacia atrás sobrepasan ligeramente la región humeral de los élitros, negras, con los seis primeros artejos de aspecto sedoso, del VI al XI paulatinamente castaño oscuros, un poco más claros hacia el extremo y mates (Figs. 2, 3). La longitud de los segmentos antenales, en $\mathrm{mm}$, y la relación "longitud/anchura máxima" son, respectivamente, 
las siguientes: I: $0.81,1.8$; II: $0.3,1.17$; III: 0.68 , 1.93; IV: $0.63,1.77$; V: $0.53,1.66$; VI: 0.51, 1.68 ; VII: $0.56,1.75$; VIII: $0.6,2$; IX: 0.58, 2.05; X: 0.58, 2.15; XI: 0.76, 3.5. Artejo I subcónico, estrechado por delante de la inserción basal, 2.12 veces más largo que ancho y 2.45 veces más largo que el siguiente; II muy corto, estrecho en la base y fuertemente ensanchado en la mitad apical, casi igual de ancho que largo; III a $\mathrm{V}$ troncocónicos, subiguales, en torno a 2 veces más largos que anchos, con la mayor anchura en la región distal, el IV ligeramente más corto que el III y el V a su vez un poco más corto que el IV; VI subcilíndrico, de longitud casi similar al $\mathrm{V}$, estrechado en los extremos y con la máxima anchura en el medio, 1.47 veces más largo que ancho; VII a X subiguales, cilíndricos, débilmente estrechados en los extremos, de lados subparalelos, entre 1.66-1.7 veces más largos que anchos, el VII y el VIII un poco más gruesos que los dos siguientes; XI alargado, 1.35 veces más largo que el anterior, de lados subparalelos excepto en la zona apical donde se estrecha débilmente para terminar en punta redondeada. Artejos antenales I a V con pilosidad negra, tumbada y recta, similar a la de los artejos del protarso, relativamente densa, al igual que el punteado en el que se inserta, que es un poco más fino que el de las sienes, en el segmento antenal I los pelos son un poco más levantados que en los tres siguientes artejos; VI a XI con pilosidad castañorojiza, cortísima, poco perceptible, aplicada contra el tegumento, a excepción de algunos pelos erectos, cortísimos y dispersos.

Pronoto negro, mate, con la superficie tegumentaria marcadamente micorreticulada, transverso y de forma subtrapezoidal (Fig. 5); longitud máxima: $2.32 \mathrm{~mm}$; anchura máxima: 3.45, a nivel de los ángulos laterales, que son obtusos, bien marcados aunque redondeados; relación anchura/longitud máximas: 1.49; relación anchura máxima/anchura en la base: 1.22; márgenes laterales netamente convergentes hacia atrás en

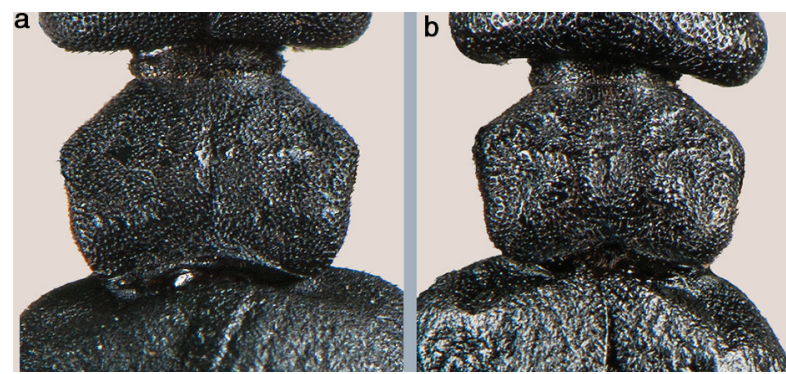

Fig. 5.- Pronotum (dorsal view) of: (a) Meloe (Eurymeloe) baamarani n. sp. (Holotipo: Tazila, Ifni, Marruecos); (b) Meloe (Eurymeloe) mediterraneus Müller, 1925 (Sierra del Haus, Tetuán, Marruecos). Longitud del pronoto en (a): $2.32 \mathrm{~mm}$; en (b) $2.41 \mathrm{~mm}$. (Fotografías: Saúl Yubero).

Fig. 5.- Pronotum (dorsal view) of: (a) Meloe (Eurymeloe) baamarani n. sp. (Holotype: Tazila, Ifni, Morocco); (b) Meloe (Eurymeloe) mediterraneus Müller, 1925 (Sierra del Haus, Tetouan, Morocco). Pronotum length in (a): $2.55 \mathrm{~mm}$; and (b): $2.75 \mathrm{~mm}$. (Photographs: Saúl Yubero). los tres cuartos posteriores y débilmente sinuados en el centro, fuertemente convergentes hacia delante en su cuarto anterior; margen posterior fuertemente escotado en arco regular en medio; margen anterior en arco poco marcado; superficie dorsal en suave declive hacia delante en el cuarto anterior y en declive abrupto hacia atrás en el cuarto posterior; zona discal un poco elevada, mostrando una depresión longitudinal mediana, de límites difusos, sin llegar a constituir un surco central, y dos depresiones anterolaterales a ambos lados de la zona elevada, poco profundas y límites difusos, que a su vez se prolongan a sendas depresiones posteriores pequeñas, muy débiles, escasamente impresas y difusas; entre la depresión anterolateral y la posterior, se observa una pequeña zona ligeramente elevada y lisa. Punteado del pronoto constituido por puntos similares a los del vértex, pequeños, muy densos y subcontiguos, que en conjunto le imprimen un aspecto suavemente subrugoso, casi uniformemente repartidos, con la menor densidad justo por detrás del margen anterior y en sendas pequeñas áreas casi impunteadas situadas cada una entre las dos leves depresiones laterales (anterior oblicua y posterior redondeada). Pilosidad pronotal negra, cortísima, similar a la de la cabeza, semitendida y curvada, siguiendo el patrón del punteado en el que se inserta; la de la mitad anterior dirigida hacia atrás y la de la región posterior dirigida hacia delante. Propleuras mate, con puntos similares a los del pronoto aunque menos impresos y menos densos, y pilosidad igualmente escasa. Mesonoto sobresaliendo escasamente de la base del pronoto, con el borde posterior subrectilíneo, con pilosidad castaño rojiza, corta y dirigida hacia atrás. Metanoto oculto en casi toda su superficie por la base de los élitros. Prosterno estrecho, poco prolongado hacia atrás, terminado en punta redondeada, con punteado similar al de las propleuras. Mesoepímeros densa y difusamente punteados, con puntos pequeños y contiguos. Placa mesosternal normalmente conformada, muy transversa, no rebordeada en su margen posterior, con una prolongación triangular entre las coxas medias terminada en punta aguda, la superficie con arruguitas longitudinales; punteado denso y poco impreso, similar al de las propleuras, con pilosidad castaño-rojiza semierecta, inclinada hacia atrás, de longitud similar a la de las coxas. Metasterno fuertemente hendido en forma de $\mathrm{U}$ abierta en el centro de su margen posterior.

Élitros de color negro y aspecto mate, montados en la base (el derecho sobre el izquierdo), fuertemente dehiscentes, relativamente alargados, llegando a la mitad anterior del abdomen, presentando una estrecha depresión basal oblicua por encima de la región humeral; tegumento microrreticulado y muy finamente rugoso, con arruguitas muy finas dispuestas de forma más o menos concéntricas a las débiles áreas foveoladas; escultura de la superficie elitral suave, constituida por unas foveolas débilmente marcadas, ondulado-redondeadas, de límites difusos 
y más o menos longitudinales; punteado apenas evidente, escaso y confundido con la rugosidad tegumentaria; pilosidad muy corta, negra, poco perceptible, con pelos semicurvados y tumbados hacia atrás, escasos y uniformemente repartidos por la superficie elitral.

Abdomen con la región dorsal de color negro mate. Terguitos abdominales con las placas esclerotizadas centrales casi indistintas, más evidentes en los tres últimos terguitos, donde son de mayor tamaño; el tegumento de las zonas no fuertemente esclerotizadas liso, el de las placas esclerotizadas finamente microrreticulado y con punteado muy fino, poco impreso, casi imperceptible y poco denso; la pilosidad de estas placas negra, muy corta y escasa, tumbada hacia atrás, casi aplicada al tegumento, sin formar mechones o agrupaciones de pelos, uniformemente repartida siguiendo el patrón del punteado en el que se inserta; el último terguito presenta en su margen posterior pelos negros unas tres veces más largos que los del resto de terguitos, rectos, más densos y agrupados en el extremo apical. Esternitos abdominales negros, de aspecto sedoso semibrillante y tegumento microrreticulado, con punteado fino, poco impreso y muy denso, confluente, que le imprime a la superficie un aspecto finamente vermiculado; pilosidad constituida por pelos negros, finos y densos, similares a los del último terguito, tumbados y aplicados contra la superficie; último esternito fuertemente escotado en el ápice y con una depresión central bien marcada.

Patas de aspecto relativamente robusto aunque largas, negras, de aspecto sedoso, con el extremo distal de los artejos del tarso tornándose castaño oscuro; tegumento muy fínamente micorreticulado; pilosidad negra, un poco más larga y gruesa que la de los esternitos abdominales, totalmente tumbada, más densa en las tibias que en los fémures. Tarsos alargados y de aspecto robusto, ligeramente más largos que las tibias, los meso- y metatarsos más largos que los protarsos, debido a que el primer segmento tarsal es netamente más corto en estos últimos; los artejos tarsales I a IV portan en su cara inferior un cepillito conspicuo de pelos negros y semierectos, un poco más cortos que los de las tibias pero más gruesos y densos, que ocupan exclusivamente entre el quinto (artejo tarsal I) y el tercio distal (tarsómeros II a IV) de los artejos tarsales, el artejo V no presenta un cepillo bien definido, únicamente algunos pelos alineados pero no apretados; pilosidad de las caras superior y laterales de los tarsómeros similar a la de las tibias, pero con pelos más cortos. Espinas de las tibias anteriores aproximadamente dos tercios la longitud del primer protarsómero, subiguales, acuminadas hacia el ápice, un poco curvadas en el extremo; espinas mesotibiales similares a las de las protibias, subiguales; espinas de las metatibias con la interna ligeramente más corta, débilmente curvada y algo más gruesa en la base que las de las mesotibias, la externa mucho más gruesa, en forma de cuchara fuertemente ensanchada, cortada en bisel en su cara superior. Uñas normalmente conformadas, castaño rojizas, débil y regularmente curvadas, con los lóbulos superior e inferior de longitud similar.

Edeago de aspecto estilizado y relativamente alargado, tanto en visión lateral como dorsal (Fig. 6); falobase 1.8 veces más larga que ancha en visión dorsal, con longitud similar a la de los parámeros, un poco más ancha que estos en visión dorsal, apenas convexa y con la máxima anchura en el medio; parámeros 3.46 veces más largos que anchos en visión lateral, más bien estrechos y alargados en visión lateral, estrechados regularmente en su tercio distal, con los lóbulos paramerales ligeramente curvados en el extremo y distalmente digitiformes, presentado lateralmente y en la mitad apical una amplia depresión alargada, poco profunda y de límites más bien
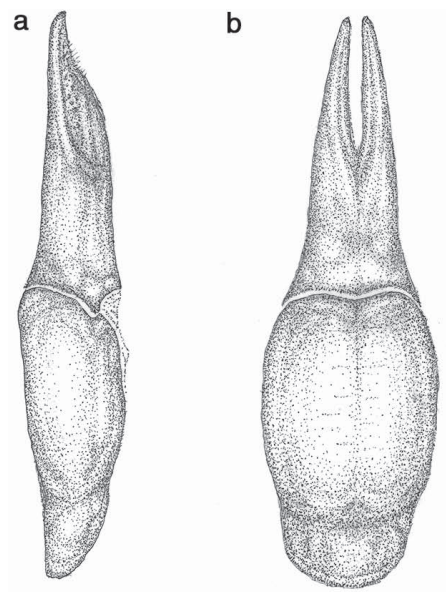

c
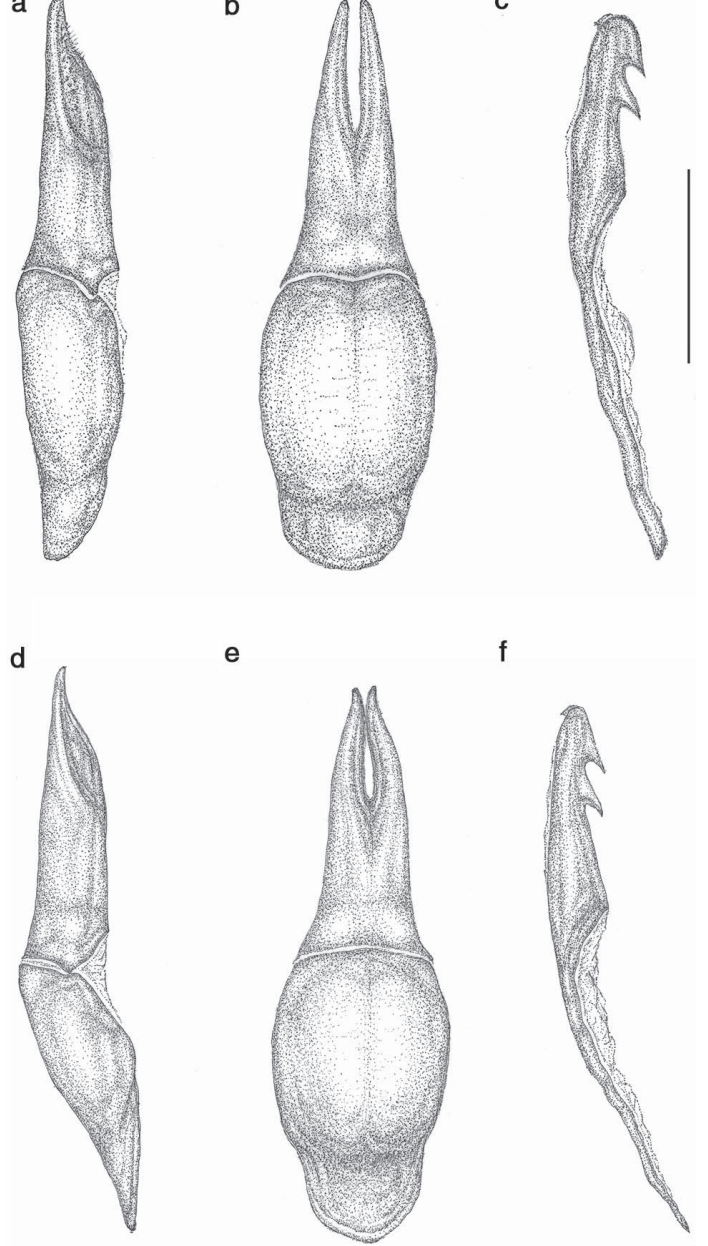

Fig. 6.- Genitalia masculina de: Meloe (Eurymeloe) baamarani n. sp. (Holotipo: Tazila, Ifni, Marruecos): tegmen en visión lateral (a) y dorsal (b), lóbulo medio en visión lateral (c); Meloe (Eurymeloe) mediterraneus Müller, 1925 (Sierra del Haus, Tetuán, Marruecos): tegmen en visión lateral (d) y dorsal (e), lóbulo medio en visión lateral (f). Escala: 0.5 mm (J.L. Ruiz del.).

Fig. 6.- Male genitalia of: Meloe (Eurymeloe) baamarani n. sp. (Holotype: Tazila, Ifni, Morocco): tegmen in lateral (a) and dorsal view (b), median lobe in lateral view (c); Meloe (Eurymeloe) mediterraneus Müller, 1925 (Sierra del Haus, Tetouan, Morocco): tegmen in lateral (d) and dorsal view (e), median lobe in lateral view (f). Scale bar: $0.5 \mathrm{~mm}$ (J.L. Ruiz del.). 
difusos, limitada dorsalmente por una estrecha y débil elevación longitudinal, esta depresión muestra en su superficie un punteado muy fino pero perceptible, en el que se insertan algunos cortísimos y a su vez muy finos micropelos blanquecinos erectos, sobre todo en las inmediaciones de los lóbulos apicales; en visión dorsal, los parámeros son relativamente estrechos, regularmente acuminados hacia la región distal, con los lóbulos paramerales redondeados en el extremo, la escotadura longitudinal-central es relativamente estrecha y prolongada un poco más de la mitad. Lóbulo medio largo y más bien robusto, sinuado dorsalmente en su mitad distal en visión lateral, muy escasamente curvado en su mitad proximal, con dos fuertes dientes ventrales subiguales, poco curvados en su extremo, cercanos entre sí, el distal próximo al ápice, que es ampliamente redondeado; gancho dorso-apical (uncus) pequeño, apenas visible (Fig. 6).

Hembra: Los ejemplares hembra presentan, en general, un tamaño corporal algo mayor que los machos (longitud total en $ᄋ$ 우 entre 13.6-19.3 mm, media $=15.38 \mathrm{~mm}, \mathrm{n}=7)$. Uno de los ejemplares hembra $(14.2 \mathrm{~mm})$ fue capturado recién emergido, con el tegumento de las patas y antenas castaño-rojizo oscuro y sin desgaste alguno. Por tal motivo, este ejemplar presenta el abdomen no distendido, con la cápsula elitral cubriéndolo casi por completo, de forma que la dehiscencia de los élitros comienza en su tercio posterior; este individuo habría de sobrepasar el rango de tamaños constatado para la especie toda vez su abdomen hubiese alcanzado un mayor volumen, pues su cabeza y pronoto son significativamente mayores que en la mayor parte de ejemplares estudiados (anchura máxima de la cabeza $=4.8 \mathrm{~mm}$; anchura máxima del pronoto $=4.1 \mathrm{~mm}$ ), con excepción de un macho de muy parecidas características. Las hembras son similares al macho, con la escultura elitral un poco más marcada que en el holotipo (foveolas y ondulaciones más aparentes). Difieren del macho principalmente por presentar un aspecto general algo más robusto, las antenas comparativamente un poco más cortas y ligeramente más robustas, y el último esternito abdominal redondeado en el ápice, sin escotadura apical y sin depresión central, uniformemente piloso. Valvífera de la hembra como en la Fig. 7.

VARIABILIDAD: La variabilidad observada en los ejemplares de la serie-tipo es relativamente amplia y afecta de manera perceptible a los siguientes caracteres: longitud total: $10.5-19.3 \mathrm{~mm}$, media $=14.26 \mathrm{~mm}$, $\mathrm{n}=18$ (页 $\sigma^{2}: 10.5-17.1 \mathrm{~mm}$, media $=13.55 \mathrm{~mm}, \mathrm{n}=11$; 우: $13.6-19.3 \mathrm{~mm}$, media $=15.38 \mathrm{~mm}, \mathrm{n}=7$ ); anchura máxima, situada entre el segundo tercio y el ápice elitral (ocasionalmente, y dependiendo de la forma adoptada por los élitros y el abdomen al secarse, en la zona media abdominal): $4.2-10.6 \mathrm{~mm}$, media $=6.77 \mathrm{~mm}$, $\mathrm{n}=18$ (ふうે: 4.2-8.9 mm, media $=6.19 \mathrm{~mm}, \mathrm{n}=11$; 오이 $6.4-10.6 \mathrm{~mm}$, media $=7.67 \mathrm{~mm}, \mathrm{n}=7$ ), si bien este carácter merístico depende tanto del grado de distensión del abdomen (y, por tanto, la separación y

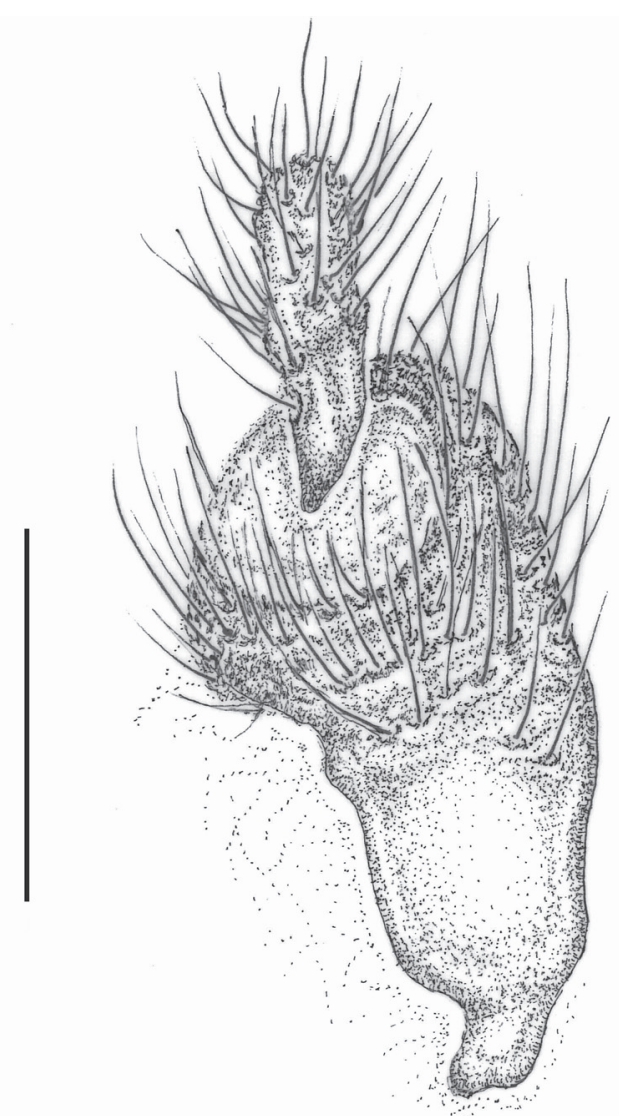

Fig. 7.- Meloe (Eurymeloe) baamarani n. sp. (paratipo hembra: Ain Aguisgal, Ifni, Marruecos): Valvifera y estilo. Escala: $0.5 \mathrm{~mm}$ (J.L. Ruiz del.).

Fig. 7.- Meloe (Eurymeloe) baamarani n. sp. (female paratype: Ain Aguisgal, Ifni, Morocco): Valvifer and stylus. Scale bar: $0.5 \mathrm{~mm}$ (J.L. Ruiz del.).

dehiscencia entre los élitros) en el momento de captura como de la retracción y forma adoptada por el mismo al secarse el ejemplar; anchura máxima de la cabeza: $2.3-5 \mathrm{~mm}$, media $=3.68 \mathrm{~mm} ; \mathrm{n}=18 \quad\left({ }_{\jmath}\right.$ : 2.3-5 mm, media $=3.4 \mathrm{~mm}, \mathrm{n}=11$; 우우 $3.25-4.8 \mathrm{~mm}$, media $=4.13 \mathrm{~mm}, \mathrm{n}=7$ ); longitud desde la sutura clípeofrontal al vértex: $1.6-3.26 \mathrm{~mm}$, media $=2.51 \mathrm{~mm}$, $\mathrm{n}=18$ (ふえへ: 1.6-3.25 mm, media $=2.35 \mathrm{~mm}, \mathrm{n}=11$; 오: $2.25-3.26 \mathrm{~mm}$, media $=2.77 \mathrm{~mm}, \mathrm{n}=7$ ); anchura máxima del pronoto: $2.2-4.35 \mathrm{~mm}$, media $=3.28 \mathrm{~mm}$,

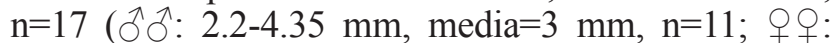
3.3-4.1 mm, media $=3.79 \mathrm{~mm}, \mathrm{n}=6$ ); longitud máxima del pronoto: $1.35-2.75 \mathrm{~mm}$, media $=2.12 \mathrm{~mm}, \mathrm{n}=17$ (ぷ $\overbrace{}^{\lambda}: 1.35-2.75 \mathrm{~mm}$, media $=1.96 \mathrm{~mm}, \mathrm{n}=11$; 우우: 2.2-2.55 mm, media $=2.42 \mathrm{~mm}, \mathrm{n}=6$ ); relación "anchura/longitud máximas" del pronoto: 1.46-1.62, media $=1.54, \mathrm{n}=17$ ( ふ઼ $\sigma^{\lambda}: 1.46-1.62$, media $=1.52, \mathrm{n}=11$; 우오: $1.5-2$, media $=1.56 \mathrm{~mm}, \mathrm{n}=6$ ); relación "anchura máxima/anchura en la base" del pronoto: 1.21-1.35,

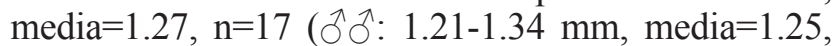
$\mathrm{n}=11$; 우우: $1.24-1.35 \mathrm{~mm}$, media $=1.31 \mathrm{~mm}, \mathrm{n}=6$ ); longitud de las antenas con ligeras variaciones, en general un poco más largas en los machos, la relación "longitud/anchura máxima" de los artejos IV a VII se expone 
en la Tabla 1; color de los artejos antenales, que en algunos individuos se torna castaño a partir del VI, al igual que las patas, y que en los ejemplares colectados tras su reciente emergencia (sin desgaste tegumentario) muestran coloración castaño-rojiza oscura, un poco más clara hacia las regiones distales; tenue depresión de la frente con ligeras variaciones en su amplitud, pero siempre muy corta y débilmente impresa, llegando a desaparecer por completo en los ejemplares de menor tamaño y, por el contrario, más marcada en los de mayor tamaño; punteado de cabeza y pronoto con cierta variación en su densidad, en general más denso y con puntos más impresos en los especímenes de mayor tamaño; depresión longitudinal media del pronoto más o menos marcada y de anchura variable, pero en general bien perceptible aunque de límites difusos, igualmente se aprecian diferencias en la amplitud e impresión de las depresiones anterolaterales, siendo más amplias y marcadas en los ejemplares de mayor tamaño; escultura y rugosidad elitral más marcada en algunos ejemplares de tamaño mayor, especialmente las ondulaciones y foveolas; punteado de los esternitos abdominales y pilosidad que en él se inserta con leves variaciones en su densidad. La genitalia masculina es muy uniforme, con ligera variación en la longitud y curvatura de los lóbulos paramerales en visión lateral, así como de la anchura de éstos y de la falobase en visión dorsal; asimismo, se detecta cierta variabilidad en el micropunteado y micropilosidad existente en las depresiones laterales de la mitad distal de los lóbulos paramerales.

No se ha observado variabilidad significativa entre los ejemplares procedentes del núcleo poblacional de Mogador (=Essaouira) y los de Ifni; no obstante, el tamaño de muestra es relativamente escaso, sobre todo de la primera localidad (5 especímenes). De igual forma, a pesar del reducido tamaño de muestra, hay ejemplares procedentes de ambas regiones con elevadas diferencias de tamaño, lo que contribuye a enmascarar posibles patrones de variabilidad interpoblacional.

DistribuCiÓN Y NOTAS AUTOECOLÓGICAS: Meloe baamarani se conoce hasta el momento de siete localidades situadas en el suroeste de Marruecos, en la costa atlántica y en sus inmediaciones, penetrando hacia el interior en el territorio de Ifni. La serie-tipo procede de dos núcleos poblacionales en principio separados entre sí unos $250 \mathrm{~km}$ en línea recta (Fig. 8): (1) el núcleo poblacional de Essaouira (antes Mogador; $31^{\circ} 30^{\prime} \mathrm{N} 9^{\circ} 44^{\prime} \mathrm{O}$, altitud: 0-25 m); (2) el de la antigua provincia española de Ifni (actual provincia marroquí de Sidi Ifni), al sur del anterior, en el Marruecos presahariano. En los territorios de Ifni, se halló en seis localidades cercanas entre sí: Adai $\left(29^{\circ} 12^{\prime} \mathrm{N}\right.$ $10^{\circ} 11^{\prime} \mathrm{O}, 450 \mathrm{~m}$ ), Tazila (=Tasila Ait Ado Bouhous/ Ahel Jader; $29^{\circ} 11^{\prime} \mathrm{N} 10^{\circ} 14^{\prime} \mathrm{O}, 515 \mathrm{~m}$ ), Taraut de Tual (=Toual; $\left.29^{\circ} 10^{\prime} \mathrm{N} 10^{\circ} 06^{\prime} \mathrm{O}, 550 \mathrm{~m}\right)$, Amasin (=Amaziz; $29^{\circ} 10^{\prime} \mathrm{N} 10^{\circ} 15^{\prime} \mathrm{O}, 270 \mathrm{~m}$ ), Ain Aguisgal (=Aguechgal, Agoujgal; $\left.29^{\circ} 11^{\prime} 16^{\prime \prime} \mathrm{N} 10^{\circ} 16^{\prime} 11^{\prime \prime} \mathrm{O}, 215 \mathrm{~m}\right)$ y Yebel Tamarrut (=Djebel Tamarnut; $29^{\circ} 12^{\prime} \mathrm{N} 10^{\circ} 01^{\prime} \mathrm{O}$; altitud máxima: $1225 \mathrm{~m}$ ). El rango altitudinal de la especie es relativamente estrecho, comprendido entre el nivel del mar y un máximo de $1225 \mathrm{~m}$.

Desde un punto de vista bioclimático, el área de ocupación (sensu UICN, 2001) de M. baamarani se integra en el ámbito del piso inframediterráneo (Benabid, 1976, 1985; Peltier, 1986), exclusivo del suroeste de Marruecos e Islas Canarias orientales (donde también se denominó infracanario; véase Rivas-Martínez, 1987), que se corresponde en el norte de África, en su mayor parte, con el sector florístico macaronésico marroquí, caracterizado por especies macaronésicas crasas y espinosas (e.g. Benabid \& Fennane, 1994; Géhu \& Biondi, 1998). Dicha área presenta un gradiente de aridez creciente en sentido norte-sur, de forma que en Essaouira el ombrotipo es semiárido superior [precipitación media anual $=295 \mathrm{~mm}$; e.g. Le Houerou (1989)] y en Sidi Ifni es árido inferior [p.m.a. $=168 \mathrm{~mm}$ en Sidi Ifni, siendo de media en torno a $150 \mathrm{~mm}$ en la totalidad del territorio provincial, e.g. Le Houerou (1989), CRI Agadir (2008)], si bien en la franja atlántica el clima es suavizado por la influencia oceánica, con un aporte importante de criptoprecipitaciones en forma de brumas y nieblas, que pueden suponer del orden de 20-50 mm anuales (Géhu \& Biondi, 1998).

Fitosociológicamente, las formaciones vegetales climácicas arborescentes y arbustivas de estas regiones, de fisionómia muy singular, se integran en el orden Acacio-Arganietalia, exclusivo del sector

Tabla 1.- Relación "longitud/anchura" máximas de los artejos antenales IV a VI de Meloe baamarani n. sp., Meloe affinis simillimus Martínez de la Escalera, 1914 y Meloe affinis apivorus Reitter, 1895.

Table 1.- Maximum length/maximum width relation in the antennal segments IV to VI of Meloe baamarani n. sp., Meloe affinis simillimus Martínez de la Escalera, 1914, and Meloe affinis apivorus Reitter, 1895.

\begin{tabular}{|c|c|c|c|c|c|c|}
\hline & \multicolumn{2}{|c|}{ Meloe baamarani $(n=13)$} & \multicolumn{2}{|c|}{ Meloe affinis simillimus $(n=26)$} & \multicolumn{2}{|c|}{ Meloe affinis apivorus $(n=2)$} \\
\hline & Rango & Media & Rango & Media & Rango & Media \\
\hline Artejo IV & $1.7-1.91$ & 1.79 & $1.29-1.48$ & 1.39 & $1.39-1.5$ & 1.44 \\
\hline Artejo V & $1.45-1.7$ & 1.6 & $1.13-1.4$ & 1.25 & $1.35-1.37$ & 1.36 \\
\hline Artejo VI & $1.57-1.72$ & 1.64 & $1.14-1.41$ & 1.24 & $1.33-1.36$ & 1.34 \\
\hline Artejo VII & $1.59-1.75$ & 1.67 & $1.09-1.38$ & 1.23 & $1.32-1.4$ & 1.36 \\
\hline
\end{tabular}




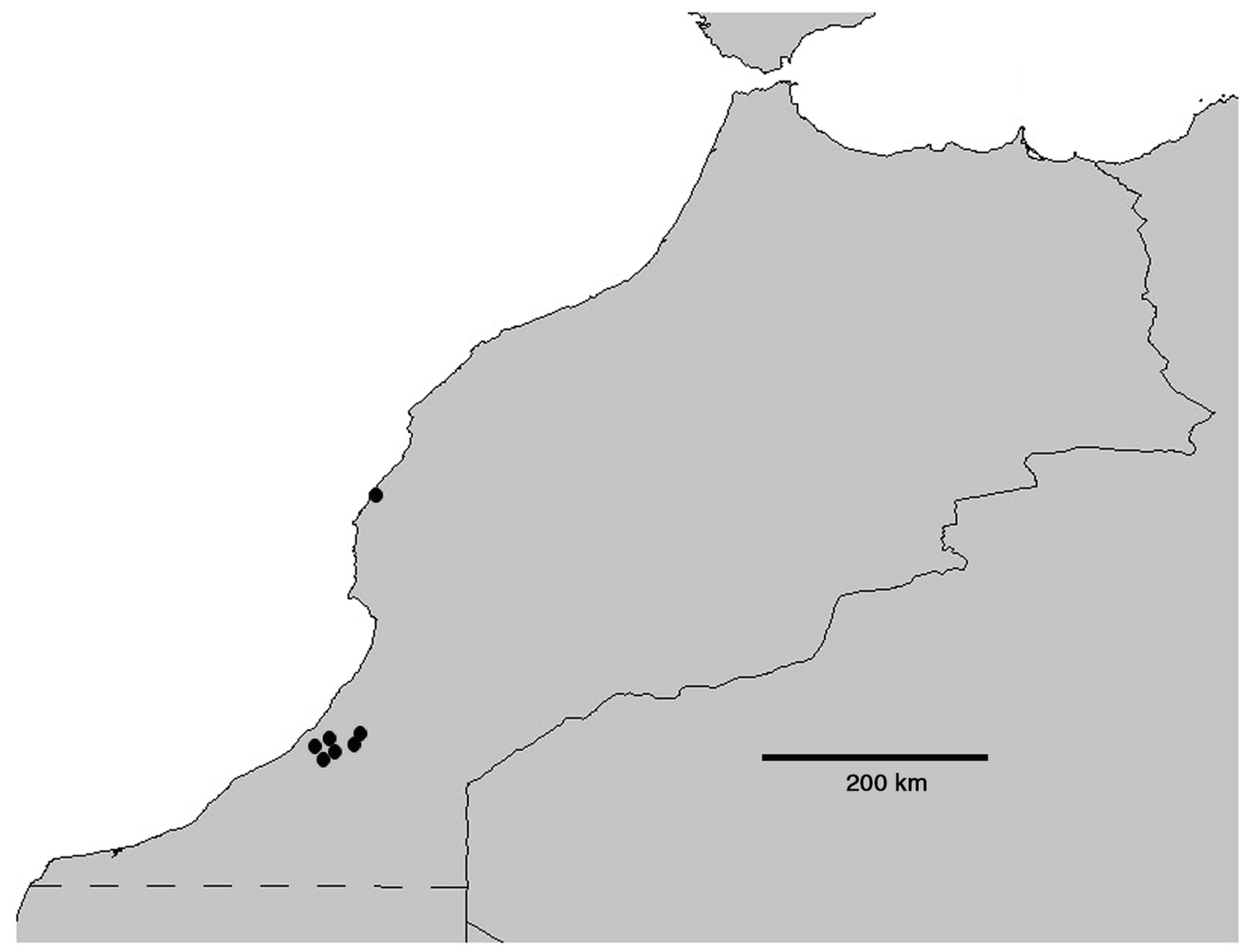

Fig. 8.- Mapa de Marruecos con las localidades conocidas de Meloe (Eurymeloe) baamarani n. sp.

Fig. 8.- Map of Morocco including known localities of Meloe (Eurymeloe) baamarani n. sp.

macaronésico inframediterráneo norteafricano, caracterizado, entre otras, por las siguientes especies: Argania spinosa (L.) Skeels, Acacia gummifera Willd., Caralluma maroccana (Hook fil.) N.E.Br., Coronilla ramossisima (Ball) Ball, Rhus pentaphylla (Jacq.) Desf., Rhus tripartita (Ucria) Grande, Periploca angustifolia Labill, Asparagus altissimus Mumby y varias especies de Euphorbia arbustivas y cactiformes (e.g. Barbero et al., 1982; Peltier, 1983, 1986; Charco, 1999). Las asociaciones vegetales mayoritarias son las denominadas Euphorbio beaumierianaeArganietum spinosae y Euphorbio echini-Arganietum spinosae (Barbero et al., 1982; Benabid \& Fennane, 1994; Charco, 1999). En líneas generales, se trata de formaciones más o menos abiertas, habitualmente de tipo sabanoide, dominadas por dos especies arbóreas propias de esta región del suroeste de Marruecos, el argán (Argania spinosa) y la acacia marroquí (Acacia gummifera), así como varias especies de Euphorbia arbustivas [E. obtusifolia subsp. regis-jubae (Webb \& Berth.) Maire, E. balsamifera Aiton] y cactiformes [E. officinarum subsp. echinus (Hooker fil. \& Cosson) Vindt, E. beaumieriana Hooker fil. \& Cosson y, muy localmente, $E$. resinifera Berger] las cuales constituyen formaciones esteparias de tipo tabaibal-cardonal, que dominan el paisaje en extensas regiones (e.g. Peltier, 1983; Benabid \& Fennane, 1994; Médail \& Quézel,
1999; Charco, 1999). Sin embargo, la sobrepresión ganadera es muy elevada y está generando la degradación de esta singular vegetación y la casi total desaparición del tapiz vegetal en amplias zonas en los últimos decenios (Benabid, 1985; Géhu \& Biondi, 1998).

Según los escasos datos de captura de los ejemplares de la serie-tipo, se colectaron exclusivamente en los meses de diciembre (en Mogador) y enero (territorios de Ifni), presentando los adultos una fenología invernal, como es habitual en otras especies del subgénero Eurymeloe (Bologna, 1991).

EtimologíA: El nombre de la especie alude a la tribu bereber (tamazight) natural de la región ifniense, los Ait Baamaran, cuyo amplio territorio tribal es en buena parte coincidente con la antiguos territorios españoles de Ifni y a su vez con la actual provincia marroquí de tal nombre.

\section{Discusión}

En el ámbito del subgénero Eurymeloe, Meloe baamarani se caracteriza por la combinación de los siguientes rasgos diagnósticos: tamaño mediano a grande (10.5-19.3 mm, media=14.26, $\mathrm{n}=18)$, coloración del cuerpo y apéndices negra, de aspecto mate; tegumento microrreticulado; pilosidad negra, corta y 
poco densa en cabeza, pronoto, élitros y tergos abdominales, sin formar mechones ni agrupaciones más o menos conspicuas; cabeza ancha y de sienes ampliamente redondeadas, sin surco longitudinal medio; antenas largas, con los artejos III a VIII netamente más largos que anchos, subcilíndricos, poco o apenas ensanchados en el extremo; pronoto transverso, entre 1.46-1.62 (media $=1.54 ; n=17$ ) veces más ancho que largo, con los lados netamente convergentes hacia atrás, sin surco mediano, pero con una depresión media relativamente amplia y de límites difusos; punteado de cabeza y pronoto densa, conformada por puntos pequeños pero bien impresos, redondeados, subconfluentes; élitros con escultura relativamente suave, formada por foveolas débilmente marcadas, ondulado-redondeadas, de límites difusos, y arruguitas finas; edeago de aspecto alargado, vaina parameral relativamente estilizada, con una región deprimida lateralmente presentando los lóbulos distales rectos y digitiformes, lóbulo medio largo y robusto, sinuado dorsalmente, con los dientes ventrales próximos al ápice.

Meloe baamarani comparte los caracteres diagnósticos del grupo I o grupo de $M$. rugosus sensu Bologna (1988), y dentro de éste en el subgrupo A, cuya especie característica es igualmente $M$. rugosus (Bologna, 1988): pilosidad del cuerpo negra o muy oscura; tegumento corporal negro, de aspecto mate, lustroso o brillante; punteado de cabeza y pronoto neto, aunque en la nueva especie lo constituyen puntos finos, aunque bien marcados; y, por último, rugosidad elitral generalmente marcada, si bien este rasgo está sujeto a amplia variabilidad intraespecífica en varias especies del grupo (Bologna, 1988, 1991), y en M. baamarani es tambien variable.

Según Bologna (1988), y asumiendo el criterio de Ruiz et al. (2010) de separar a M. saharensis en un subgrupo independiente, el subgrupo A o de M. rugosus agruparía a 13 especies, si bien como apunta este autor (Bologna, 1988, 1991) algunas de ellas, como M. affinis, M. baudii o M. fernandezi, se incluyen aquí de forma provisional y a efectos prácticos, pues morfológicamente son bastante diferentes de las demás y las relaciones filogenéticas entre ellas y con las del resto del subgrupo están por dilucidar. En este sentido, Di Giulio et al. (2013) señalan que $M$. affinis debe constituir un grupo independiente del de M. rugosus.

Del total de taxones específicos integrados en el subgrupo podemos diferenciar, según su distribución geográfica, dos conjuntos de especies bien separados (ver Bologna, 1988: 243): (1) un grupo oriental, de especies asiáticas y (2) un conjunto paleártico occidental (dos especies se hallan también en la macaronesia) en el cual se integra M. baamarani. Así, entre las primeras encontramos seis especies exclusivas de la región Transcaucásica, Asia central y Oriente medio (con excepción de una especie, M. glazunovi Pliginskij, 1910, que se extiende por el oeste hasta
Grecia; Bologna, 1994b, 2008), de las que apenas se tiene información (e.g. Escherich, 1896; Reitter, 1895; Pliginskij, 1910, 1911; Kaszab, 1958, 1978; Bologna, 1988, 1991, 1994b, 2008; Kolov, 2003). Entre las segundas, una es endémica de las Islas Canarias, M. fernandezi, y las otras seis presentan una distribución europea y/o mediterránea occidental más o menos amplia: M. affinis, M. mediterraneus, M. ganglbaueri, M. rugosus, M. apenninicus y M. baudii, de ellas, únicamente las dos primeras se hallan en el norte de África.

Meloe rugosus, especie de amplia distribución, conocida desde Inglaterra a la depresión del Caspio, y $M$. apenninicus, exclusiva de Sicilia y la práctica totalidad de la Península italiana (e.g. Bologna, 1988, 1991, 2008, com. pers. 2014; Pezzi, 2009), se separan claramente de $M$. baamarani, entre otros caracteres, por presentar aquellas el tegumento corporal negro brillante (azabache), en lugar de netamente mate; la cabeza con un fuerte surco centro-longitudinal prolongado desde la sutura clípeo-frontal hasta el vértex, ausente en la nueva especie; pronoto más transverso en estas dos especies europeas, presentando un fuerte surco longitudinal mediano (véase Bologna, 1988: p. 292 , figs. $12-13$, p. 294 , figs. $40-41$; 1991: p. 313 , fig. 108) inexistente en la nueva especie (en su defecto, una depresión central no asurcada); punteado de cabeza y pronoto significativamente más grueso e impreso, con puntos casi el doble de diámetro en las dos primeras y élitros con rugosidad y vermiculación netamente más marcados en éstas.

Meloe ganglbaueri presenta una distribución que se extiende desde Italia hasta Siria (Bologna, 1988, 1991, 2008) y ha sido citada puntualmente del sur de la Península Ibérica (Ruiz \& Ávila, 1993); según Bologna (1988, 1991, 1994b) y Di Giulio et al. (2013) las antiguas citas del Magreb (e.g. Cros, 1943) serían erróneas y la presencia de la especie en el norte de África requeriría confirmación. $M$. ganglbaueri se diferencia a primera vista de $M$. baamarani por presentar la primera toda la pilosidad de la región dorsal del cuerpo castaño amarillenta (dorada), con conspicuos mechones de pelos de idéntico color en el margen posterior de los terguitos abdominales, el punteado de cabeza y pronoto marcadamente más grueso y profundo, presencia de un surco longitudinal medio en la región frontal de la cabeza y pronoto de aspecto más transverso (Bologna, 1988: p. 255-257, p. 294, fig. 42; 1991: 318-321, p. 320, fig. 111a-f).

Meloe baudii, distribuido por Italia peninsular, Sicilia, Cerdeña y Croacia (e.g. Bologna, 1988, 1991, 2008) y posiblemente sinónimo de M. glazunovi (M.A. Bologna, com. pers. 2014), se segrega fácilmente de M. baamarani por presentar aquella las antenas netamente más cortas, con los artejos IV a VI apenas alargados, casi igual de ancho que largos (este carácter acerca $M$. baudii a $M$. affinis, que también presenta las antenas relativamente cortas, con dichos artejos muy similares, cortos y engrosados en el extremo); además, 
M. baudii presenta el tegumento corporal negro brillante, los puntos de la cabeza gruesos y el pronoto transverso con el disco plano, entre otros caracteres diferenciales (Bologna, 1988: 262-264, p. 292, fig. 16, p. 293 , fig. 29 , p. 294 , fig. 44 ; 1991: 309-311, p. 308 , fig. $105 \mathrm{f}-\mathrm{k})$.

Meloe fernandezi se separa con claridad de $M$. baamarani, entre otros rasgos, por presentar la primera un rango de tamaño mayor (13-24 mm frente a 10.5-17.1 mm); el tegumento corporal brillante en la primera; el pronoto netamente menos transverso, sinuado en los lados, con la escultura del tegumentaria muy particular, presentando estrías y arrugas lineales paralelas entre sí, onduladas, formando algunos remolinos; y élitros con rugosidad fina pero fuertemente marcada, con las rugosidades brillantes y en zig-zag longitudinales (Pardo Alcaide, 1951; García et al., 1993). M. fernandezi es un endemismo canario conocido de las islas de La Palma y Tenerife (Pardo Alcaide, 1951; Bologna, 1988, 1994a; Bologna \& Marangoni, 1990; García et al., 1993; Machado \& Oromí, 2000; García, 2008).

A pesar de la complejidad intraespecífica de $M$. affinis, ésta se diferencia claramente de M. baamarani, entre otros, por la conjunción de los siguientes rasgos, comunes a los dos taxones marroquíes (M. a. simillimus y $M$. a. apivorus): tegumento corporal sedoso en $M$. affinis, mate en la nueva especie; antenas comparativamente más cortas en $M$. affinis, con los artejos troncocónicos y más cortos y anchos en esta especie (véase Bologna, 1988: 293, figs. 30-32, 1991: p. 328, fig. 114b), más largas, con los artejos subcilíndricos y más estrechos en M. baamarani, así, la relación "longitud/anchura máxima" en los segmentos antenales IV a VII es significativamente mayor en M. baamarani (véase Tabla 1); pronoto netamente más ancho y transverso en $M$. affinis, de manera que la relación "anchura/ longitud máximas" en $M$. a. simillimus oscila entre 1.66-1.95 (media=1.77; $\mathrm{n}=26$ ), en $M$. a. apivorus entre $1.77-1.81$ (media=1.79; $\mathrm{n}=2$ ) y en $M$. baamarani entre 1.46-1.62 (media $=1.54 ; \mathrm{n}=17)$; depresiones anterolaterales de la superficie pronotal impresas, más o menos redondeadas y bien perceptibles en $M$. baamarani, apenas o escasamente marcadas y trasversales en $M$. affinis; punteado de cabeza y pronoto mucho más denso y fino en la nueva especie. La subespecie nominal es todavía más distinta, pues además de compartir los rasgos diferenciales indicados, presenta la cabeza más ancha y truncada posteriormente, y el punteado de la cabeza y pronoto muy escaso, apenas perceptible (véase Bologna, 1988: 266-273, p. 292, fig. 17, p. 294, fig. 45; 1991: 326-330, p. 328, fig. 114a-f).

La especie morfológicamente más cercana a $M$. baamarani, con la que comparte una facies muy parecida, es M. mediterraneus. Ambas especies se separan por los siguientes rasgos: aspecto general distinto (Figs. 2, 3), con el tegumento corporal, sobre todo en cabeza, pronoto, élitros y tergos abdominales, lustroso (satinado) o sedoso en $M$. mediterraneus, claramente mate, sin apenas brillo en M. baamarani, con un microrreticulado neto en esta última, que en la primera es imperceptible y en su caso aparece un micropunteado muy fino y disperso; cabeza (Fig. 4) en M. mediterraneus con un profundo y largo surco frontal longitudinal, que se extiende casi desde la sutura clípeo-frontal hasta el vértex, sobrepasando ampliamente el nivel del margen posterior de los ojos (con cierta variación en su longitud y profundidad, dependiendo fundamentalmente del tamaño del ejemplar), mientras que M. baamarani no presenta dicho surco, a lo sumo, en los ejemplares de mayor tamaño (observado en cinco ejemplares), aparece una débil y corta depresión longitudinal en el centro de la frente (un poco por detrás del nivel del borde posterior de los ojos), de límites difusos; pronoto de aspecto distinto en ambas especies (Fig. 5): más transverso (relación "anchura/longitud máximas" entre 1.46-1.62; media $=1.54 ; n=17)$, ofreciendo un aspecto hexagonal, y con los márgenes laterales posteriores netamente convergentes, oblicuos, en M. baamarani (relación "anchura máxima/anchura en la base" entre 1.21-1.35; media=1.27; $\mathrm{n}=17$ ), siendo en $M$. mediterraneus un poco menos transverso (relación "anchura/longitud máximas" entre 1.30-1.47; media $=1.42 ; n=36$ ), con los márgenes laterales posteriores subparalelos, débilmente convergentes (relación "anchura máxima/anchura en la base" entre 1.03-1.17; media $=1.12 ; n=36$ ), de forma que el pronoto muestra un aspecto subcuadrado; disco del pronoto en M. baamarani con una depresión centro-longitudinal relativamente ancha, de límites difusos pero bien perceptible, y sendas depresiones anterolaterales también netas, por el contrario, en $M$. mediterraneus el disco del pronoto no presenta dicha depresión patente, sólo en ocasiones una débil zona deprimida, y las depresiones anterolaterales son menos marcadas; punteado de cabeza y pronoto muy diferente: en $M$. baamarani los puntos son más finos, entre un tercio y casi la mitad de diámetro que en $M$. mediterraneus, donde el punteado es netamente más grueso, ofreciendo un aspecto subvermiculado, con densidad mucho menor en la cabeza y mayor en pronoto en la primera especie respecto a la segunda; pilosidad de la cabeza y pronoto la mitad de larga en $M$. baamarani y muy tendida, de forma que es menos perceptible que en $M$. mediterraneus; esternitos abdominales manifiestamente más brillantes, con punteado rasposo también más abundante, subrugoso, en $M$. mediterraneus (véase Bologna, 1988: 258-262, p. 292 , fig. 15 , p. 293 , fig. 28 , p. 294 , fig. 43 ; 1991 : 322-325, p. 320, fig. 111g-1).

La genitalia masculina presenta diferencias significativas entre ambas especies (Fig. 6): parámeros con la depresión lateral de la región distal más profunda y con límite dorsal más neto, asurcado, con punteado muy débil, escaso y difuso, sin pilosidad perceptible en $M$. mediterraneus, siendo en $M$. baamarani esta depresión más débil, pero con un punteado pilífero 
patente, más denso, con micropelos erectos y blanquecinos que se extienden hacia los lóbulos distales; en visión dorsal, los lóbulos parámerales son más alargados y estrechos en $M$. baamarani y la hendidura longitudinal media en más ancha y alargada en esta especie; lóbulo medio más robusto, redondeado en el extremo distal, sinuado dorsalmente en visión lateral y con el diente ventral distal cercano al ápice en $M$. baamarani, mientras que en $M$. mediterraneus es acuminado en la región apical, recto y con dicho diente más alejado del ápice.

Meloe mediterraneus presenta una amplia distribución por toda la cuenca mediterránea, incluida macaronesia (Islas Canarias, Madeira, Porto Santo y Desertas), llegando por el este hasta la región del Cáucaso, Turquía oriental e Irán (Bologna, 1988, 1991, 1994a, 2008, 2009; Machado \& Oromí, 2000). En Marruecos se encuentra distribuida por las regiones septentrional, central y occidental del país, desde Tánger al norte, y Taza al este, hasta el Anti Atlas en el sur (Bled Akhas; Kocher, 1938, 1956), excepcionalmente en montaña en el Medio Atlas (DayetAhoua, $1400 \mathrm{~m}$ ), si bien son escasos los registros concretos; no ha sido señalada de los territorios de Ifni. M. mediterraneus y M. baamarani no parecen encontrase en simpatría. No obstante, es probable la presencia de poblaciones de M. mediterraneus en la región de Essaouira e incluso más al sur, en el extremo septentrional del área de ocupación (sensu UICN, 2001) de M. baamarani, pues esta localidad atlántica se sitúa al norte de los contrafuertes suroccidentales del Anti Atlas.

Por otro lado, teniendo en cuenta el carácter forético de las larvas de primer estadío de las especies de Eurymeloe, lo que facilita la colonización e intercambio faunístico con medios insulares (e.g. Bologna, 1988, 1991; Bologna et al., 1989; Bologna \& Marangoni, 1990), así como las condiciones ambientales y ecológicas de la franja inframediterránea semiárida-árida del suroeste de Marruecos en la que habita $M$. baamarani (donde se desarrolla la denominada vegetación "macaronésica"; Médail \& Quézel, 1999), muy semejantes a las de las Islas Canarias orientales (pisos infra- y termomediterráneo canarios; véase e.g. Rivas-Martínez et al., 2002), no es descartable la presencia de esta especie en las islas de Lanzarote, Fuerteventura, Gran Canaria e incluso en ambientes semiáridos-secos de Tenerife.

Por último, quisiéramos llamar la atención sobre el inapreciable patrimonio científico que constituyen las extensas colecciones de historia natural, especialmente las entomológicas, reunidas por los Martínez de la Escalera, Manuel (padre) y Fernando (hijo), en el noroeste de África (Ruiz \& García-París, 2011). Martín Albaladejo (2011) resalta el inmenso valor como "vivero de datos" de tales colecciones, que aún actualmente (sic) "continúan ofreciendo información relevante a los entomólogos interesados por la entomofauna de esa área". Sin duda, la presente aportación taxonómica es una pequeña muestra de ello e ilustra, una vez más, las novedades que todavía nos depara el ingente legado de los Martínez de la Escalera.

\section{Agradecimientos}

A Marco A. Bologna (Universidad Roma Trè), por su ayuda y colaboración, tanto facilitándonos el estudio de su colección como por sus comentarios. A Saúl Yubero, por la realización de las fotografías que ilustran este trabajo y por su intensa colaboración en el trabajo de campo. También agradecemos a Ernesto Recuero, Iñigo Martínez-Solano, José Luis Zapata, Pablo Barranco, Rafael Yus, José M. Barreda, Ramón Sánchez, Thomas Gazurek, Miguel de Benito, Mercedes París, Manuel Sánchez Ruiz, Nohemí Percino, Gonzalo García Martín, Ángel Montes, Reda Garmute y Jorge Gutiérrez Rodríguez, la información o ejemplares aportados o su compañía en los muestreos. A Mercedes París, por su inestimable ayuda en la consulta de las colecciones históricas de Entomología del MNCN. A Jesús Benzal, que nos facilitó el estudio de la colección de la EEZA (CSIC-Almería). A Gloria Masò por facilitarnos el estudio de material de Museu de Zoologia de Barcelona (MZB). A Antoine Mantilleri por permitir la revisión de las colecciones históricas del Muséum National d'Histoire Naturelle de París (MNHN). A Pedro Oromí por facilitarnos la visita a la colección de la Universidad de La Laguna (UL). A Pepe Fernández por su ayuda en cuestiones taxonómicas y editoriales. Parte de los muestreos y la infraestructura básica para la realización de este trabajo ha sido aportada por el proyecto CGL2010-15786 (Ministerio de Ciencia y Tecnología, España). La visita a la colección del MNHN de París ha sido realizada gracias al apoyo del Proyecto SYNTHESYS (http://www.synthesys.info/) financiado por la "European Community Research Infrastructure Action" dentro del programa FP7 “Capacities”. Agradecemos al Instituto de Estudios Ceutíes el apoyo prestado en todo momento.

\section{Referencias}

Alcobendas, M., Ruiz, J. L., Settanni, C. \& García-París, M., 2008. The taxonomic status of Euzonitis haroldi (Heyden, 1870) (Coleoptera: Meloidae) inferred from morphological and molecular data. Zootaxa, 1741: 59-67.

Barbero, M., Benabid, A., Quézel, P., Rivas-Martínez, S. \& Santos, A., 1982. Contribution à l'étude des AcacioArganietalia du Maroc sud-occidental. Documents phytosociologiques (n.s.), 6: 311-338.

Benabid, A., 1976. Étude phytoécologique, phytosociologique et sylvopastorale de la tétraclinaie de l'Amsittene. Thèse $3^{\text {ème }}$ cycle, Université Aix-Marseille III. Marseille. $155 \mathrm{pp}$.

Benabid, A., 1985. Les écosystèmes forestiers, préforestiers et presteppiques du Maroc: diversité, répartition biogéographique et problèmes posés par leur aménagement. Forêt méditerranéenne, 7(1): 53-64.

Benabid, A. \& Fennane, M., 1994. Connaissances sur la végétation du Maroc: Phytogéographie, phytosociologie et séries de végétation. Lazaroa, 14: 21-97.

Bolívar, I., 1935. Apuntes para la fauna entomológica de Ifni (Ortópteros). Eos, 11: 395-426, láms. 17-21.

Bologna, M. A., 1988. Note su Eurymeloe e revisione delle specie euromediterranee del gruppo rugosus (Coleoptera, Meloidae). Fragmenta Entomologica, 20(2): 233-301. 
Bologna, M. A., 1991. Fauna d'Italia. XXVIII. Coleoptera Meloidae. Calderini. Bologna. xiv+541 pp.

Bologna, M. A., 1994a. Meloidae from Canary and other macaronesian islands (Coleoptera). Miscel-lània Zoològica, [1992], 16: 73-80.

Bologna, M. A., 1994b. I Meloidae della Grecia (Coleoptera). Fragmenta Entomologica, 25 (Supplemento): 1-119.

Bologna, M. A., 2008. Meloidae. In: I. Löbl \& A. Smetana (eds.). Catalogue of Palaearctic Coleoptera. Vol. 5. Tenebrionoidea. Apollo Books. Stenstrup: 370-412.

Bologna, M. A., 2009. The Meloidae (Coleoptera) of Libya: an annotated catalogue and description of three new species. Annales de la société entomologique de France (n.s.), 45(3): 345-364. http://dx.doi.org/10.1080/003792 71.2009 .10697620$.

Bologna, M. A., Aloisi, G. \& Marangoni, C., 1989. Nuove osservazioni su Eurymeloe Reitter e descrizioni di larve di I stadio (Coleoptera, Meloidae). Bulletin et Annales de la Société Royale Belge d'Entomologie, 125: 67-75.

Bologna, M. A. \& Marangoni, C., 1990. Dispersal, dispersion and phoresy in the blister beetle fauna (Coleoptera, Meloidae) of eastern Mediterranean and other islands. Atti dei Convegni "Biogeographical aspects of insularity”, Accademia Nazionale dei Lincei, 85: 345-366.

Bologna, M. A. \& Pinto, J. D., 2002. The Old World genera of Meloidae (Coleoptera): a key and synopsis. Journal of Natural History, 36(17): 2013-2102. http://dx.doi. org/10.1080/00222930110062318.

Borchmann, F., 1917. Pars 69. Meloidae, Cephaloidae. In: E. Schenkling (ed.). Coleopterorum Catalogus auspiciis et auxilio W. Junk. Junk. Berlin. 208 pp.

Charco, J., 1999. El bosque mediterráneo en el norte de África. Biodiversidad y lucha contra la desertificación. Agencia Española de Cooperación Internacional. Madrid. 370 pp.

CRI Agadir, 2008. Monographie de la province de Sidi Ifni. Centre Régional d'Investissement Souss Massa Dráa. 16 pp. Disponible en: http://www.cri-agadir.ma/french/ monographie sidi-ifni.pdf [consultado el 17/09/2013].

Cros, A., 1934. Le Meloe affinis Lucas var. setosus Escherich ses moeurs, sa larve primaire. Bulletin de la Société d'Historie naturelle de l'Afrique du Nord, 21 : 88-104, 2 pls.

Cros, A., 1936. Le Meloe affinis Lucas Var. setosus Escherich. Étude biologique. Bulletin de la Société d'Historie naturelle de l'Afrique du Nord, 27: 185-196, 1 pl.

Cros, A., 1939. Les Meloidae des possesions françaises de l'Afrique du Nord. Étude biogéographique. Bulletin et Annales de la Société entomologique de Belgique, 79: 247-265.

Cros, A., 1943. Considérations genérales sur les espèces de Meloe du groupe «rugosus» Marsh. Observations biologiques sur le $M$. ganglbaueri Apfelbeck et le $M$. mediterraneus Muller. Annales des Sciences naturelles, Zoologie, 5(11): 61-77.

Di Giulio, A., Sciotti, A. \& Bologna, M. A., 2013. Revision of the first instar larvae of Meloe, subgenera Eurymeloe and Coelomeloe, with new descriptions and a key to the species (Coleoptera: Meloidae). Italian Journal of Zoology, 80(2): 242-254.
Escherich, K., 1890. Revision der behaarten Meloë-Arten der alten Welt. Wiener Entomologische Zeitung, 9: 87-96.

Escherich, K., 1896. Meloiden-studien. IV. Theil. Wiener Entomologische Zeitung, 15: 27-30.

García, R., 2008. Distribución de la familia Meloidae (Coleoptera) en la Isla de La Palma. Revista de Estudios Generales de la Isla de La Palma, [2007], 3: 363-380.

García, R., Ortega, G. \& Pérez, J. M., 1993. Insectos de Canarias. Excmo. Cabildo Insular de Gran Canaria. [1992]. Las Palmas de Gran Canaria. 418 pp.

García-Figueras, T., 1941. Santa Cruz del Mar PequeñaIfni-Sáhara. La acción de España en la costa occidental de África. Ediciones Fe. Madrid. 350 pp.

García-París, M., Alcobendas, M., Buckley, D. \& Wake, D. B., 2003. Dispersal of viviparity via hybridization in Salamandra as inferred from nuclear and mitochondrial DNA phylogenetic discordances. Evolution, 57: 129-143. http://dx.doi.org/10.1111/j.0014-3820.2003.tb00221.x.

García-París, M., Parra-Olea, G. \& Wake, D. B., 2008. Description of a new species of the Bolitoglossa subpalmata group (Caudata: Plethodontidae) from Costa Rica. Herpetological Journal, 18: 23-31.

García-París, M. \& Ruiz, J. L., 2005. Bibliografía y registros Ibero-Baleares de Meloidae (Coleoptera) publicados hasta la aparición del "Catálogo sistemático de los Coleópteros observados en la Península Ibérica, Pirineos propiamente dichos y Baleares" de J. M. de la Fuente (1933). Graellsia, 61(2): 225-255. http://dx.doi. org/10.3989/graellsia.2005.v61.i2.20.

García-París, M. \& Ruiz, J. L., 2011. Capítulo 9. Las cantáridas y aceiteras (Coleoptera: Meloidae) en la obra de Manuel Martínez de la Escalera. In: C. Martín Albaladejo \& I. Izquierdo Moya (eds.). Al encuentro del naturalista Manuel Martínez de la Escalera (1867-1949). Monografías del Museo Nacional de Ciencias Naturales. Consejo Superior de Investigaciones Científicas. Madrid: 173-205, láms. 9-14.

Géhu, J. M. \& Biondi, E., 1998. Nature et limites de quelques végétations littorales de type macaronésien sur les côtes sud occidentales du Maroc. Acta Botanica Barcinonensia, 45 (Homenatge a Oriol de Bolòs): 439-453.

Kaszab, Z., 1958. Die Meloiden Afghanistan (Coleoptera). Acta zoologica Academiae scientiarum Hungaricae, 3: 245-312.

Kaszab, Z., 1978. Neue Meloiden aus Asien, Afrika und Südamerika (Coleoptera). Acta zoologica Academiae scientiarum Hungaricae, 24: 331-342.

Kocher, L., 1938. Localisations nouvelles ou intéressantes de coléoptères marocains. Bulletin de la Société de Sciences Naturelles du Maroc, 18: 77-118.

Kocher, L., 1953. Localisations nouvelles ou intéressantes de Coléoptères marocains. Travaux de l'Institut Scientifique Chérifien, 7: 1-142.

Kocher, L., 1956. Catalogue commenté des Coléoptères du Maroc. V. Héteromères (Tenebrionides excepts). Travaux de l'Institut Scientifique Chérifien (série Zoologie), 10: 1-107.

Kolov, S. V., 2003. [Taxonomic composition of blisterbeetles (Coleoptera, Meloidae) of Kazakhstan]. Tethys Entomological Research, 8: 157-168. [En ruso, con resumen en inglés]. 
Le Houerou, H. N., 1989. Classification écoclimatique des zones arides (s.1.) de l'Afrique du Nord. Ecologia mediterranea, 15(3/4): 95-144, 7 tabs.

Machado, A. \& Oromí, P., 2000. Elenco de los Coleópteros de las Islas Canarias. Instituto de Estudios Canarios, Monografía 70. La Laguna. 306 pp.

Mader, L., 1927. Meloidae. In: A. Winkler (ed.). Catalogus Coleopterorum regionis palaearcticae, vol. II. Winkler. Wien: 851-888.

Martín Albaladejo, C., 2011. Capítulo 11. Martínez de la Escalera en el noroeste de África: la huella de sus exploraciones entomológicas. In: C. Martín Albaladejo \& I. Izquierdo Moya (eds.). Al encuentro del naturalista Manuel Martínez de la Escalera (1867-1949). Monografías del Museo Nacional de Ciencias Naturales. Consejo Superior de Investigaciones Científicas. Madrid: 221-241.

Martínez de la Escalera, M., 1914. Los Coleópteros de Marruecos. Trabajos del Museo Nacional de Ciencias Naturales, serie Zoológica, 11: 1-553.

Martínez de la Escalera y Carrasco, G., 2011. Capítulo 2. Fernando Martínez de la Escalera: aproximación al legado humanista de Manuel Martínez de la Escalera. En: C. Martín Albaladejo \& I. Izquierdo Moya (eds.). Al encuentro del naturalista Manuel Martínez de la Escalera (1867-1949). Monografías del Museo Nacional de Ciencias Naturales. Consejo Superior de Investigaciones Científicas. Madrid: 45-74.

Médail, F. \& Quézel, P., 1999. The phytogeographical significance of S.W. Morocco compared to the Canary Islands. Plant Ecology, 140: 221-244.

Müller, G., 1925. Sulle Meloë europee del grupo rugosus. Studi Entomologici, Racolta lavori entomologia sistematica, 1(1): 21-25.

Pardo Alcaide, A., 1951. Estudios sobre Meloidae. III. Una nueva especie de Meloe de la isla de Tenerife y comentarios sobre algunos meloideos de la citada isla. Eos, Revista Española de Entomología, 25: 249-255.

Peltier, J. P., 1983. Les séries de l'arganeraie steppique dans le Sous (Maroc). Ecologia Mediterranea, 9(1): 77-88.

Peltier, J. P., 1986. L'étage de végétation inframéditerranéen dans le Sous. Documents phytosociologiques (n.s.), 10(1): 437-454.

Peyerimhoff, P. de., 1926. Meloidae. 94-95. In: J. SainteClaire Deville \& P. de Peyerimhoff. Nouveaux Coléoptères du Nord-Africain. Cinquante-cinquième note. Faune du Grand-Atlas marocain (suite). Bulletin de la Société entomologique de France, [1926]: 93-96.

Peyerimhoff, P. de., 1949. Études et descriptions des coléoptères marocains II. Bulletin de la Société de Sciences naturelles et physiques du Maroc, [1945-1947], 25-27: 248-308.

Pezzi, G., 2009. Meloe (Eurymeloe) apenninicus Bologna, 1988 nella Riserva Naturale Integrale di Sasso Fratino (Forlì-Cesena): una specie nuova per gli Apennini settentrionali. Quaderno di Studio e Notizie di Storia Naturale della Romagna, 28: 33-35.

Pina, J., 2011. Capítulo 19. Manuel y Fernando Martínez de la Escalera por el noroeste de África: expediciones entre 1905 y 1912. In: C. Martín Albaladejo \& I. Izquierdo Moya (eds.). Al encuentro del naturalista Manuel Martínez de la Escalera (1867-1949). Monografías del
Museo Nacional de Ciencias Naturales. Consejo Superior de Investigaciones Científicas. Madrid: 365-391.

Pinto, J. D. \& Selander, R. B., 1970. The bionomics of blister beetles of the genus Meloe and a classification of the New World species. Illinois Biological Monographs, 42: 1-222. http://dx.doi.org/10.5962/bhl.title.50239.

Pliginskij, V. G., 1910. Deux espèces nouvelles du genre Meloë Linn. (Coleoptera, Meloidae). Revue Russe d'Entomologie, 10: 170-172.

Pliginskij, V. G., 1911. Notice sur un caractère chez les representant du genre Melö̈. Revue Russe d'Entomologie, 11: 44-47.

Reitter, E., 1895. Bestimmungs-Tabellen der europaïschen Coleopteren Meloidae. I Theil: Meloini. 32. Verlag des Verfassers. Paskau. 13 pp.

Rivas-Martínez, S., 1987. Memoria del mapa de las series de vegetación de España 1:400.000. ICONA. Madrid. $268 \mathrm{pp}$.

Rivas-Martínez, S., Díaz, T. E., Fernández-González, F., Izco, J., Loidi, J., Lousa, M. \& Penas, A., 2002. Vascular plant communities of Spain and Portugal. Addenda to the syntaxonomical checklist of 2001. Part I. Itinera Geobotanica, 15(1): 5-432.

Ruiz, J. L. \& Ávila, J. M., 1993. Contribución al conocimiento de los Meloidae (Coleoptera) en el sur de la Península Ibérica. Boletín de la Asociación española de Entomología, 17(2): 141-148.

Ruiz, J. L. \& García-París, M., 2009. Descripción de una especie nueva de Meloe Linnaeus, 1758 del subgénero Eurymeloe Reitter, 1911 (Coleoptera, Meloidae) del norte de Marruecos. Graellsia, 65(2): 91-109. http://dx. doi.org/10.3989/graellsia.2009.v65.i2.144.

Ruiz, J. L., Bologna, M. A. \& García-París, M., 2010. Taxonomía y distribución de Meloe (Eurymeloe) saharensis Chobaut, 1898 (Coleoptera, Meloidae), con nuevas sinonimias y primeros registros para Europa y la Macaronesia. Graellsia, 66(1): 85-96. http://dx.doi.org/ 10.3989/graellsia.2010.v66.015.

Ruiz, J. L. \& García-París, M., 2011. Reflexiones sobre la labor científica de M. Martínez de la Escalera. En: C. Martín Albaladejo \& I. Izquierdo Moya (eds.). Al encuentro del naturalista Manuel Martínez de la Escalera (1867-1949). Monografías del Museo Nacional de Ciencias Naturales. Consejo Superior de Investigaciones Científicas. Madrid: 243-254.

UICN (Unión Internacional para la Conservación de la Naturaleza), 2001. Categorías y Criterios de las Listas Rojas de la UICN: Versión 3.1. Comisión de Supervivencia de Especies de la UICN. UICN, Gland \& Cambridge. $\mathrm{ii}+33 \mathrm{pp}$.

Wiley, E. O., 1978. The evolutionary species concept reconsidered. Systematic Zoology, 27: 17-26. http:// dx.doi.org/10.2307/2412809.

Wiley, E. O., 1981. Phylogenetics. The theory and practice of phylogenetic systematics. University of Kansas, Lawrence \& John Wiley and sons, New York. $439 \mathrm{pp}$.

Wiley, E. O. \& Mayden, R. L., 2000. The evolutionary species concept. En: Q. D. Wheeler \& R. Meier (eds.). Species Concept and Phylogenetic Theory: A Debate. Columbia University Press. New York: 70-92. 
Apéndice 1.- Material examinado del subgénero Eurymeloe. Las siglas utilizadas son las siguientes: MNCN: Museo Nacional de Ciencias Naturales, CSIC (Madrid, España); EEZA: Estación Experimental de Zonas Áridas, CSIC (Almería, España); MZB: Museu de Zoologia (Barcelona, España); MNHN: Muséum National d'Histoire Naturelle (París, Francia); MB: Colección Marco A. Bologna (Universidad Roma Tre, Roma, Italia); UL: Universidad de La Laguna (Tenerife, España); JLR: Colección J. L. Ruiz (Ceuta, España); RS: Colección Ramón Sánchez (España); JLZ: Colección José Luis Zapata (Madrid, España); TG: Colección Thomas Gazurek (Polonia).

Appendix 1.- Material examined of subgenus Eurymeloe. The acronyms used are as follows: MNCN: Museo Nacional de Ciencias Naturales, MNCN-CSIC (Madrid, Spain); EEZA: Estación Experimental de Zonas Áridas, CSIC (Almería, Spain); MZB: Museu de Zoologia (Barcelona, Spain); MNHN: Muséum National d'Histoire Naturelle (Paris, France); MB: Marco A. Bologna collection (Roma Tre University, Roma, Italy); UL: La Laguna University (Tenerife, Spain); JLR: José L. Ruiz collection (Ceuta, Spain); RS: Ramón Sánchez collection (Spain); JLZ: José Luis Zapata collection (Madrid, Spain); TG: Thomas Gazurek collection (Poland).

\section{Meloe (Eurymeloe) rugosus Marsham, 1802}

SUECIA: ESCANIA: 1 ejemplar: "Lund" / "Meloe rugosus" / "Muséum Paris, 1930 Coll. Sicarol" / "Meloe (Eurymeloe) rugosus Marsham 1802 David Král det. 2013" (MNHN).

REPUBLICA CHECA: 1 ejemplar: "S. Boehmerwald, Tanzer" / "Meloe (Eurymeloe) rugosus Marsham, M. Bologna det. 19" (CB).

REPUBLICA ESLOVACA: 1 ejemplar: "Slovak. Cepelal Trenen" / "Meloe rugosus" (MNHN).

GRECIA: 1 ejemplar: "Grecia, Peloponesso, Taigeto, Katafighion, m 1650, 3.11.[1]978, A. Vigna leg.” (CB).

\section{Meloe (Eurymeloe) apenninicus Bologna, 1988}

ITALIA: SICILIA: 1 ejemplar: "Sicilia, ME, M. Nebrodi, Biviere di Cesarò, 27-X-1975, G. Carpaneto leg." / "Paratypus, Meloe (Eurymeloe) apenninicus n. sp., M. Bologna det. 1986" [etiqueta roja, manuscrita e impresa] (JLR, ex MB). CALABRIA: 2 ejemplares: "Calabria, Sila, M. Gariglione, 1300, 4-V-1975, R. Mourglia leg." (JLR, ex MB).

\section{Meloe (Eurymeloe) baudii Leoni, 1907}

ITALIA: PIEMONTE: 1 ejemplar: "Pecetto, TO, 18-3-[1]972, G. Bolfa leg.” (CB).

Meloe (Eurymeloe) mediterraneus Müller, 1925 ARGELIA: 1 ejemplar: "Jos [?] d'Oran, Bhicher" / "rugosus Mrs." [manuscrita de Escalera] (MNCN).

MARRUECOS: 1 ejemplar: "Tánger, M. Escalera" / "Meloë rugosus Marsh., Pardo Alcaide det. 1951" (MNCN); 1 ejemplar: "Beni Msuar" [etiqueta manuscrita de Escalera] (MNCN); 1 ejemplar: "Beni Msuar, M. Escalera" (MNCN); 1 ejemplar: "Tanger, M. Escalera" / "Beni Msuar, M. Escalera" (MNCN); 1 ejemplar: "Tanger, M. Escalera" / "Beni Msuar, M. Escalera" / "Meloe rugosus Marsh. Pardo Alcaide det. 1951" / "Meloe (Eurymeloe) mediterraneus (G. Müller, 1925), J.L. Ruiz det. 2006" (MNCN); 1 ejemplar: "Tanger, M. Escalera" / "M. rugosus Mars." [manuscrita de Escalera] / "Meloe rugosus Marsh. Pardo Alcaide det. 1951" (MNCN); 1 ejemplar: "Tanger" [manuscrita de Escalera] (MNCN); 1 ejemplar: "Tanger" / "Muséum Paris, Coll. H. Marmottan 1914" / "Meloe (Eurymeloe) mediterraneus J. Müller, M. Bologna det. 1992" (MNHN); 2 ejemplares: "Sierra del Haus, Agnan-Borya, prov. Tetuán, 30S0279006, 3961110, 375 m., 23-XI-2008, J.L. Ruiz leg." (JLR).

ESPAÑA: ALBACETE: 1 ejemplar: "Alcaraz" (MNCN); 1 ejemplar: "Casas de Lázaro" (MNCN); 1 ejemplar: "Molinicos" (MNCN); 1 ejemplar: "Nerpio" (MNCN); 1 ejemplar: "Ossa de Montiel, Ermita de San Pedro, Lagunas de Ruidera, 6-II-2010, M. Sánchez Ruiz, M. París, N. Percino Daniel \& M. GarcíaParís leg." (MNCN, conservado en alcohol). ALICANTE: 1 ejemplar: "Puerto de la Carrasqueta: 4-XI-2002, bajo piedra,
R. Sánchez leg." (RS). ALMERÍA: 1 ejemplar: "Bco. del Horcajo, T.M. Laujar, Almería, 30SWF093997, 1.150 m., 24-X-1988, P. Barranco leg." (JLR); 1 ejemplar: "Paterna del Río, Sa Nevada, 30SWF043976, 1.194 m., 30-XII-1989, P. Barranco leg." (JLR). ÁVILA: 1 ejemplar: "Casillas, Colonia Embalse de los Morales, 20-X-1990, R. Sánchez leg." (RS); 1 ejemplar: "Dehesa de Piedralaves: 17-X-1992, R. Sánchez leg." (RS); 2 ejemplares: "Dehesa de Piedralaves: 1-XI-1992, R. Sánchez leg." (RS). BADAJOZ: 1 ejemplar: "Fregenal de la Sierra - Higuera la Real, $38^{\circ} 09^{\prime} 46.3^{\prime \prime} \mathrm{N}-06^{\circ} 41^{\prime} 47.5^{\prime \prime} \mathrm{O}, 704 \mathrm{~m}, 4-\mathrm{I}-2013$, E. Recuero \& M. García París leg." (MNCN, conservado en alcohol); 1 ejemplar: "Monasterio de Tentudia, 9-XI-2010, M. París \& M. Sánchez Ruiz leg." (MNCN, conservado en alcohol). BARCELONA: 1 ejemplar: "Barcelona, 25-II-1967" (EEZA). BURGOS: 1 ejemplar: "Sa [Sierra] Obarenes" / "Meloe (Eurymeloe) mediterraneus, M.A. Bologna det. 1992" (MNCN). CÁCERES: 1 ejemplar: "Ovejuela, 2-IV-2010, J. Gutiérrez-Rodríguez leg." (MNCN, conservado en alcohol). CÁDIZ: 11 ejemplar: "Algeciras, Arias leg." (MNCN); 11 ejemplar: "San Roque, XI-1968, A. Cobos leg." (EEZA); 1 ejemplar: "Puerto de las Cabras, Sierra de Ojén, A. Cobos leg." (EEZA); 2 ejemplares: "Los Barrios, Cádiz, 30-I-1982, J. de Ferrer leg." (JLR); 1 ejemplar: "Crrtra. Ubrique-Puerto Gáliz, 7-XII-1989, J. M. Ávila leg." (JLR); 1 ejemplar: "Sa del Cabrito,

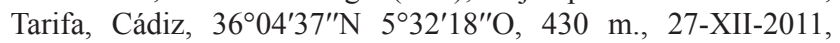
S. Yubero leg." (JLR); 1 ejemplar: "Km 27 Carretera E5/N340 de Vejer a Conil, 11-XII-1995, R. Sánchez leg.” (RS); 2 ejemplares: "Km 3.7 Carretera de Alcalá de los Gazules a Paterna: 14-XII-1995, R. Sánchez leg. (RS); 1 ejemplar: "Tarifa / Algeciras, 16-I-2010, T. Gazurek leg." (TG). CEUTA (N. de África): 1 ejemplar: "Parcela 114, Benzú, Ceuta, 13-XII-2004, J.L. Ruiz leg." (JLR); 1 ejemplar: "Bco. Central, Benzú, Ceuta, 35 $35^{\circ} 44^{\prime}{ }^{\prime} \mathrm{N} 5^{\circ} 22^{\prime} 26^{\prime \prime} \mathrm{O}, 85 \mathrm{~m}$, 31-I-2014, J.L. Ruiz leg." (JLR); 2 ejemplares: "Cuatro Caminos, Mte. Hacho, Ceuta, 20-XI-1995, J.L. Ruiz leg.” (JLR). CIUDAD REAL: 2 ejemplares: "Casas de las Sotillas, 15-II-1985, J.L. Zapata leg." (MNCN); 1 ejemplar: "El Acebuchar, 13-II-1986, J.L. Zapata leg." (MNCN); 2 ejemplares: Pozuelo de C. [Calatrava]" (MNCN). CÓRDOBA: 1 ejemplar: "Jardín Brillante, Córdoba capital, 24-II-1985, J.M. Hidalgo leg." (JLR). GRANADA: 3 ejemplares: "Granada, Taboada leg." (MNCN); 1 ejemplar: "Granada, 4-IV-01 [1901]" / "Meloe (Eurymeloe) mediterraneus, M.A. Bologna det.1992" (MNCN); 1 ejemplar: "Nacimiento Río Castril, Sierra de Castril, 23-XI-1993, J.L. Ruiz leg." (JLR); 1 ejemplar: "Cortijo Nacimiento, Sierra de Castril, 12-VII-1991, J.L. Ruiz leg. (hallado cadáver empalado por Lanius sp.)" (JLR); 1 ejemplar: "Turrillas, T.M. Almuñecar, 10-I-1998, J.de la Peña leg." (JLR); 1 ejemplar: "Padul, Granada, 22-XI-1980, J.M. Ávila leg.” (JLR); 1 ejemplar: "Zújar, Granada, 14-IV-1982, J.M. Ávila leg." (JLR); 1 ejemplar: "Cenes, Granada, 27-X-1980, J.M. Ávila

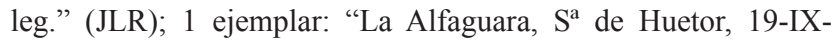
1991, R. Salado leg." (JLR); 1 ejemplar: "El Chorrillo, Alfaguara, 
Granada, 26-I-1984, J.M. Ávila leg.” (JLR); 1 ejemplar: "Silla del Moro, Granada capital, 23-IV-1972, R. Yus leg." (JLR); 4 ejemplares: "Silla del Moro, Granada capital, 18-XI-1973, R. Yus leg." (JLR); 1 ejemplar: "Crrtra. Ventas de Zafarraya-Alhama, Llanos de Jútigar, $36^{\circ} 59^{\prime} 37^{\prime \prime} \mathrm{N} 4^{\circ} 05^{\prime} 54^{\prime \prime} \mathrm{O}, 975$ m., 27-XII-2008, J.L. Ruiz leg." (JLR). GUADALAJARA: 1 ejemplar: "Espinosa de H. [Henares], Paz leg." / "Col. del Sr. Pérez Arcas" (MNCN). HUELVA: 1 ejemplar: "Cala, C. Bolívar leg." (MNCN); 1 ejemplar: "Arroyo Plamencia, Linares de la Sierra, Huelva, 22-X-2010, J.M. Barreda leg." (JLR). JAÉN: 2 ejemplares: "Pto. Las Palomas, P.N. Cazorla, Jaén, 6-XII-2002, M. Baena leg.” (JLR). MÁLAGA: 1 ejemplar: "Málaga, C. Bolívar leg." (MNCN); 1 ejemplar: "Málaga, E. Gros leg." (MNCN); 1 ejemplar: "Málaga, J. Ardois leg." (MNCN); 2 ejemplares: "Málaga, vivero, XII-928, Vázquez leg." (MNCN); 2 ejemplares: "Cortijo Marchamona, T.M. Periana, Málaga, 30S0395431, 4090057, 880 m., 29-XI-2008, J.L. Ruiz leg." (JLR y MNCN); 2 ejemplares: "Boquete de Zafarraya (vertiente Málaga), T.M. Alcaucín, 30S0399219, 4089382, 4-I-2009, J.L. Ruiz leg.” (JLR); 2 ejemplares: "Sedella, Área recreativa-Pista hacia muladar, $\mathrm{S}^{\mathrm{a}}$ Tejeda, Málaga, $36^{\circ} 52^{\prime} \mathrm{N} 4^{\circ} 02^{\prime} \mathrm{O}$, 850 m., 29-III-2013, J.L. Ruiz leg.” (JLR). MADRID: 1 ejemplar: "2 km al SO de Valdemanco, 22-X-2010, I. Martínez-Solano \& E. Recuero leg." (MNCN, conservado en alcohol); 1 ejemplar: “3 km al SO de Colmenar de Oreja, $40^{\circ} 04^{\prime} 31.88^{\prime \prime} \mathrm{N}-3^{\circ} 24^{\prime} 19.78^{\prime \prime} \mathrm{O}$, 28-III-2010, M. García-París \& R. Garmute leg." (MNCN, conservado en alcohol); 1 ejemplar: "Las Matas, $720 \mathrm{~m}, 40^{\circ} 33^{\prime} 21^{\prime \prime} \mathrm{N}$ $3^{\circ} 54^{\prime} 54^{\prime \prime} \mathrm{O}, 25-\mathrm{II}-2014$, M. de Benito leg. (MNCN, conservado en alcohol). MURCIA: 1 ejemplar: "Cartagena, 10-I-1903" (MNCN); 2 ejemplares: "Cartagena" / "Meloe (Eurymeloe) mediterraneus, M.A. Bologna det. 1992" (MNCN); 9 ejemplares: "Mazarrón" (MNCN). SEVILLA: 2 ejemplares: "Cazalla de la $\mathrm{S}^{\mathrm{a}}$ [Sierra], Quirós leg." (MNCN); 1 ejemplar: "Sierra de San Pablo, Montellano, Sevilla, 30-XI-2002, J.M. Barreda leg.” (JLR). TOLEDO: 1 ejemplar: "Pto. del Milagro, 11-XII-1991, J.L. Zapata leg." (MNCN). ZARAGOZA: 1 ejemplar: "El Frago, 11-X-2010, M. García-París \& G. García Martín leg." (MNCN, conservado en alcohol). ISLAS BALEARES: 1 ejemplar: "Mallorca Palma" / "Muséum Paris 1946, J. Ma Palau" / "Meloe (Eurymeloe) mediterraneus J. Müller, M. Bologna det. 1985" (MNHN). ISLAS CANARIAS: 1 ejemplar: "Canarias, La Gomera, I-69. J. Gil leg." (MZB); 1 ejemplar: "Iles Canaries, Fuerteventura, Ch. Alluaud 1890" / "Meloe nudus W." (MNCN).

PORTUGAL: ALGARVE: 1 ejemplar: "Sa Alcaria do Cume, 27-XI-1997, J. Fdez. Cardenete leg." (JLR). BEJA: 1 ejemplar: "São Martinho das Amoreiras, 37³6'57.4"N-08²7'57.3"O, 200 m, 4-I-2013, E. Recuero \& M. García-París leg." (MNCN, conservado en alcohol).

ITALIA: SICILIA: 1 ejemplar: "Sicilie" / "M. rugosus" / "Muséum Paris 1930 Coll. Sicard" / "Meloe (Eurymeloe) mediterraneus J. Müller, M. Bologna det. 1996” (MNHN). CERDEÑA: 1 ejemplar: "Sardegna, 500 m, Bruncals, Candelazzu, 24-X-85, C. Meloni leg." (JLR).
FRANCIA: VAR: 1 ejemplar: "Toulon"/ "Muséum Paris 1939, Coll. H. Sietti" / "Meloe (Eurymeloe) mediterraneus J. Müller, 1925 David Král det. 2013" (MNHN); 1 ejemplar: "Le Beausset, H. Sietti" / "Muséum Paris 1939, Coll. H. Sietti" / "Meloe (Eurymeloe) mediterraneus J. Müller, 1925 David Král det. 2013" (MNHN). PYRENÉES-ATLANTIQUES: 1 ejemplar: "Valleé d'Ossau Bas. Pyr. 1938” / "ex Coll. Dailli” / "Meloe (Eurymeloe) mediterraneus J. Müller, 1925 David Král det. 2013” (MNHN).

Meloe (Eurymeloe) affinis simillimus Martínez de la Escalera, 1914 MARRUECOS: MEDIO ATLAS: 1 ejemplar (sintipo): "Glaui", "M. simillimus Esc.", "Sintipo", "MNCN Cat. Tipos No 1914" (MNCN); 1 ejemplar: "Glaui”, "clavicornis Esc." (MNCN); 2 ejemplares: "Timadit" (MNCN); 4 ejemplares: "Azrou" (MNCN); 1 ejemplar: "Azrou, 12-4-1925" (MNCN); 3 ejs: "Timadit, 14-4-1923" (MNCN); 2 ejemplares: "Timhadit, 21-5-1923" (MNCN); 3 ejemplares: "Ain Leuh, 16-4-1923” (MNCN); 1 ejemplar: "Ras el Má, 16-4-1923" (MNCN); 2 ejemplares: "Ifrane, M. Atlas, V-1990, G. Blanca leg." (JLR); 1 ejemplar: "Ifrane, 29-VI-1993, J.M. Ávila leg." (JLR); 1 ejemplar: "Col du Zad, Kenifra, Medio Atlas, 1-IV-1988, J.M. Ávila leg.” (JLR); 1 ejemplar: "Crrtra. Azrou-Mischlifen km.7, 3 km. antes de Mischlifen, 1900 m., M. Atlas, 1-IV-1988, Bastazo \& Vela, leg." (JLR); 1 ejemplar: "Yebel Hayyam, Bekrit, Medio Atlas, 27-II-1997, F. Delgado leg." (JLR); 1 ejemplar: "Es Saheb, Medio Atlas, 4-V-1997, F. Delgado leg." (JLR); 4 ejemplares: "2 km al S. de Cedro Goureau, Ifrane, $33^{\circ} 24^{\prime} 57^{\prime \prime} \mathrm{N} 5^{\circ} 09^{\prime} 15^{\prime \prime} \mathrm{O}, 7-\mathrm{V}-2010$, J.L. Ruiz leg." (JLR y MNCN, los depositados en esta institución, 2 ejemplares, conservados en alcohol).

\section{Meloe (Eurymeloe) affinis apivorus Reitter, 1895}

MARRUECOS: ALTO ATLAS: 1 ejemplar: "Haut Atlas, Maroc, Tizi n’Test, 1900 m, 8-IV-1989, G. Sama leg." (MB); 1 ejemplar: "Alto Atlante, Tizi n'Test, Mouldikht, 2000 m, 18-V-1981, A. Gianasso leg." (MB).

\section{Meloe (Eurymeloe) ganglbaueri Apfelbeck, 1905}

ITALIA: LAZIO: 5 ejemplares: "Necrópolis etrusca, Tarquinia, prov. Viterbo, 14-II-2009, M. García-París \& J.L. Ruiz leg.” (JLR y MNCN, los depositados en esta institución, 3 ejemplares, se encuentran conservados en alcohol); 1 ejemplar: "Monti Tolfa, Allumieri, Palano, Pian Gallo, 25-V-85, F. Comandini leg." (JLR); 1 ejemplar: "Monti Tolfa, Allumieri, Palano, 25-IV-83, F. Comandini leg." (JLR).

\section{Meloe (Eurymeloe) fernandezi Pardo Alcaide, 1951}

ISLAS CANARIAS: LAS PALMAS: 1 ejemplar: "Las Lomadas, 30-XII-1982, R. García B. leg." (UL); 1 ejemplar: "Las Lomadas, 15-II-1998, P. Oromí leg." (UL); 6 ejemplares: "Las Lomadas, 2-III-2010, E. Recuero \& M. García-París leg." (MNCN). 